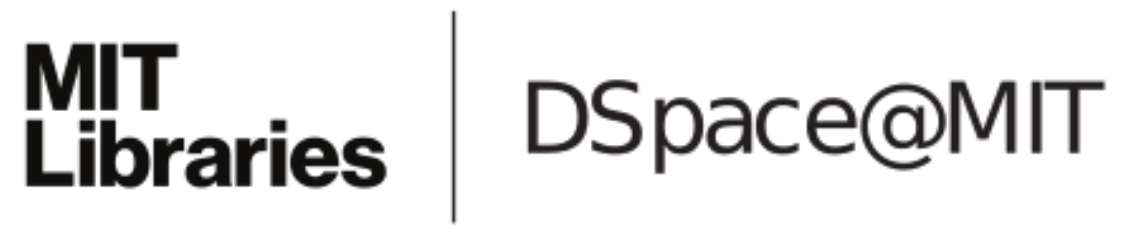

\author{
MIT Open Access Articles
}

Colloquium: Failure of molecules, bones, and the Earth itself

The MIT Faculty has made this article openly available. Please share how this access benefits you. Your story matters.

Citation: Buehler, Markus J. and Sinan Keten. "Colloquium: Failure of molecules, bones, and the Earth itself." Reviews of Modern Physics 82.2 (2010): 1459-1487. (c) 2010 The American Physical Society

As Published: http://dx.doi.org/10.1103/RevModPhys.82.1459

Publisher: American Physical Society

Persistent URL: http://hdl.handle.net/1721.1/58599

Version: Final published version: final published article, as it appeared in a journal, conference proceedings, or other formally published context

Terms of Use: Article is made available in accordance with the publisher's policy and may be subject to US copyright law. Please refer to the publisher's site for terms of use. 


\title{
Colloquium: Failure of molecules, bones, and the Earth itself
}

\author{
Markus J. Buehler* \\ Laboratory for Atomistic and Molecular Mechanics, Department of Civil and Environmental \\ Engineering, Massachusetts Institute of Technology, 77 Massachusetts Avenue, \\ Room 1-235A\&B, Cambridge, Massachusetts 02139, USA; \\ Center for Materials Science and Engineering, Massachusetts Institute of Technology, \\ 77 Massachusetts Avenue, Cambridge, Massachusetts 02139, USA; \\ and Center for Computational Engineering, Massachusetts Institute of Technology, \\ 77 Massachusetts Avenue, Cambridge, Massachusetts 02139, USA
}

\section{Sinan Keten}

Laboratory for Atomistic and Molecular Mechanics, Department of Civil and Environmental Engineering, Massachusetts Institute of Technology, 77 Massachusetts Avenue, Room 1-235A\&B, Cambridge, Massachusetts 02139, USA

(Published 10 May 2010)

\begin{abstract}
Materials fail by recurring rupture and shearing of interatomic bonds at microscopic, molecular scales, leading to disintegration of matter at macroscale and a loss of function. In this Colloquium, the state-of-the-art of investigations on failure mechanisms in materials are reviewed, in particular focusing on atomistic origin of deformation and fracture and relationships between molecular mechanics and macroscale behavior. Simple examples of fracture phenomena are used to illustrate the significance and impact of material failure on our daily lives. Based on case studies, mechanisms of failure of a wide range of materials are discussed, ranging from tectonic plates to rupture of single molecules, and an explanation on how atomistic simulation can be used to complement experimental studies and theory to provide a novel viewpoint in the analysis of complex systems is provided. Biological protein materials are used to illustrate how extraordinary properties are achieved through the utilization of intricate structures where the interplay of weak and strong chemical bonds, size and confinement effects, and hierarchical features play a fundamental role. This leads to a discussion of how even the most robust biological material systems fail, leading to diseases that arise from structural and mechanical alterations at molecular, cellular, and tissue levels. New research directions in the field of materials failure and materials science are discussed and the impact of improving the current understanding of materials failure for applications in nanotechnology, biotechnology, medicine as well as the built environment.
\end{abstract}

DOI: 10.1103/RevModPhys.82.1459

PACS number(s): 62.20.Hg, 87.14.E-, 62.20.mm, 87.19.R-

\section{CONTENTS}

I. Introduction

II. The Physics of Failure at Different Scales

III. Atomistic and Molecular Modeling of Materials Failure

A. Conventional and reactive force fields

B. Multiscale simulation techniques

IV. Case Studies: Failure of Materials, from Nano to Macro

A. Failure of the Earth's crust: Earthquakes

B. Failure of bone: Fracture processes in injury

C. Failure at molecular level: H-bond rupture in protein materials

D. Failure of hierarchical materials: Putting it all together

V. Materials Failure Phenomena in the Context of Disease

VI. Discussion

*FAX: +1-617-324-4014. mbuehler@MIT.EDU

Acknowledgments

1483

References

1483

1459

1461

1464

1466

1468

1470

1470

1472

1474

1477

1479

1482

The rupture of the Earth's crust in earthquakes, collapse of buildings, and the fracture of bones during injury are catastrophic phenomena with a common underlying theme: The breakdown of the basic constituents of any material ultimately leads to the failure of its overall structure and intended function. Failure and deformation of engineering materials has been studied extensively and has changed our world by enabling the design of complex structures and advanced devices. Eras of civilization are marked by our developing understanding and use of these materials, starting with Stone Age, Bronze Age, Industrial Age, leading into the information technology (IT) and the Space Age with the development of semiconductors and light-weight polymer materials. The most recent innovations have occurred in the field of nanotechnology and nanoscience, where in particular cross-disciplinary interactions with the biological sciences present an enormous opportunity for 
innovative basic research and also technological advancement. Such advances could enable us to provide engineered materials and structures with properties that resemble those of biological systems, in particular the ability to self-assemble, to self-repair, to adapt and evolve, and to provide multiple functions that can be controlled through external cues. However, despite significant advancements in the study of biological materials in the past decade, the fundamental physics of many phenomena in biology continue to pose substantial challenges with respect to model building, experimental studies, and simulation. Specifically, the understanding of the mechanisms of failure in biological systems remains a major issue, in particular in the context of breakdown of tissue in disease states, the failure of biological components due to injuries, and the ability of biological systems to mitigate adverse effects of damage through self-healing mechanisms. Because of our lacking ability to engineer biological materials, we also remain hindered in our ability to mass produce and utilize these materials for daily life applications, through consumer products, medical devices, and large-scale systems in aerospace, defense, and building technologies. The hierarchical bottom-up design approach in biology, from the level of genes (DNA) to proteins, to tissues, organs, and organisms, originates at the molecular scale and requires a bottom-up description from a fundamental perspective. For this reason, approaches rooted in physics that consider the structure-process-property paradigm of materials science are a powerful means to investigate the properties of biological materials.

This Colloquium is focused on discussing the origin and mechanisms of materials failure. The starting point for discussing failure in materials is coming up with a rigorous definition for failure. Simply put, failure occurs when an engineered or natural component suddenly loses its capacity to provide the service it was originally designed for, rendering it either impossible or risky to use. The key factor here is that this loss is often sudden yet significant, and that it occurs during the expected lifetime of the component. With regards to this simple explanation, failure in structural materials and structures occurs when the load bearing capacity of the designed system is significantly reduced or completely lost due to a sudden, generally unforeseen development. In the case of natural or biological systems, the definition remains the same, and is characterized by a sudden loss of function. This could be or instance the sudden rupture and slipping of the tectonic plates in an earthquake, which affects the ground's ability to provide stable foundation for the built environment. An excerpt from Darwin's The Voyage of the Beagle, describing an earthquake he experienced in Chile, illustrates how we perceive the failure of the Earth's crust:

"A bad earthquake at once destroys our oldest associations: the earth, the very emblem of solidity, has moved beneath our feet like a thin crust over a fluid;- - one second of time has created in the mind a strange idea of insecurity, which hours of reflection would not have produced."

In a simple view of failure, there are typically two aspects to the problem; the designed material system and service conditions (for example, mechanical loads). Materials deform when they are subjected to loads; this may or may not be observable by the naked eye but is definitely observable in the microscopic world, as the molecular bonds stretch, rotate, and shear, which provides the basis for a material's ability to change its shape. When the loads exceed a certain limit, bonds begin to rupture, initiating the atomistic mechanism for failure. Depending on the properties of interatomic bonds and the structure of the material at the nanoscale, failure will occur through a variety of atomistic mechanisms, leading to, for instance, brittle or ductile failure, or very slow onset of failure as observed in creep and fatigue. Once the governing unit processes such as cracks, dislocations, diffusional mass transport, molecular unwinding, or sliding propagate through the material, they become observable at the macroscale and lead to failure of a larger component in the system, for instance, a beam in the case of a building collapse, bone in case of an injury, or the breakdown of cells in genetic disease.

It is quite interesting from a historical perspective to consider how the field of fracture and failure evolved since the earliest scientific works in the field. While the foundation of the field is attributed to the work of Griffith (1921) and Irwin (1957) in developing analytical methods for studying fracture of solids, many other historical notables have shown interest in the field, such as Leonardo da Vinci, who studied scaling of the failure strength of iron wires as a function of their length and flaw presence. Although his study was not definitive due to the making and quality of the wires at that time, he was way ahead of his time in his insight to hypothesize an inverse proportionality of length and strength, such that shorter wires are stronger for a given thickness (Bazant, 1999). Galileo Galilei also studied the strength of wires as a function of thickness, and applied the same concept to testing of marble columns to conclude that the strength depends on the cross-sectional area of the column yet not on the length, thereby providing the intellectual basis for the concept of stress, defined as force per unit area. Mariotte, a court engineer at the time of Louis XIV of France, developed the concept of failure strain to describe fracture strength of pressurized vessels, and also realized that larger structures are likely to fail more easily due to the increased probability of having a weakened zone. Some of these ideas further developed after the Industrial Revolution, but no significant scientific development was achieved until Griffith proposed that the physical basis for strength limit of materials is governed by flaws in the materials, such as voids, cracks, and other structural imperfections.

Following this breakthrough, the 20th century marked the rapid development of the field of fracture mechanics, where the analytical treatment of glass, ceramics, metals, polymers, thin films, and most recently, biological materials and tissues was developed. The most recent expan- 
sion of the concepts of fracture models towards biological materials and biological systems still bases on the fundamental concept that flaws in the material ultimately control their overall strength; and the question of how biological systems are capable of tolerating and healing such flaws has received particular interest from the physics community. A failure of a biological organism to function is often related to a catastrophic response of a system to existing or newly emerging flaws, such as genetic mutations, protein misfolding, or the production of foreign material in tissues.

As pointed out before, identifying properties of materials is only half of the task; predicting service conditions is an equally demanding undertaking. Many of the colossal failures in engineering practice or in medicine are rooted in extreme loading conditions or a combination of factors (where each of which alone would not be catastrophic) that were not anticipated in the design process or under typical evolutionary constraints. Examples of such failures are many, and they have shaped our understanding of materials design for increasingly safer practices and have driven our scientific curiosity to elucidate the physical principles of life. The wind induced collapse of the Tacoma Narrows Bridge, or massive seismic activities such as the Northridge earthquake in California provided us with clues about how dynamic nature of loading can lead to unforeseen failures in large structures. Brittle fracture of the Liberty Ships during World War II illustrated how low temperatures in cold climates can literally cause ships to snap like matchsticks. Fatigue induced failure of the Comet airplanes, and later the Aloha Airlines Boeing 737 jets, illustrated the importance of corrosion and cyclic loading due to pressure changes. Failure of tissues and organs in genetic or infectious disease are other vivid examples that illustrate the significance of failure in the context of life sciences, with severe impacts on our very human existence. The central modern day challenges involve the comprehensive understanding failure across a vast range length and time scales-encompassing materials that will last for years in the harsh, unearthly conditions of the far reaches of space, or on the quite contrary within the smallest scales of human physiology as part of an effort to develop "invisible" implants that will monitor, regulate, and repair biological processes at molecular precision.

The framework of understanding failure provides us with the foundation to ask fundamental questions about the multiscale behavior of materials under extreme loading conditions and under varying outside constraints. One of the long-term goals of this research field is to develop a new engineering paradigm that encompasses the seamless analysis and design of structures and materials, starting from the molecular level. The work that roots in first addressing fundamental concepts of materials and structures may lead to the development of a new set of tools that can be applied, together with advanced synthesis methods, to select, design, and produce a new class of materials, similar to the approaches used today in computer aided design of buildings, cars, and machines, but now applied to engineer the fundamental molecular makeup of materials.

The purpose of this Colloquium is to discuss specifically the state-of-the-art theoretical and computational modeling of failure in a variety of materials, and to showcase the relevance of these methods to real life physical phenomena observed through novel experimental techniques that range in accuracy and resolution from single atoms to large geographical scales. We intend to shed light on the future prospects of research in this field by presenting an overview of established as well as recently developed methods in modeling complex materials phenomena, through a selection of case studies on multiscale atomistic and theoretical modeling. Section II discusses theoretical models that explain the physical mechanisms that lead to failure at the atomic scale; Sec. III is dedicated to review atomistic modeling techniques that have been used to illustrate these mechanisms; Sec. IV presents case studies on earthquakes, bone fracture, and failure of protein molecules; Sec. V discusses materials failure in the context of disease, and Sec. VI concludes by discussing the state-ofthe-art research and its directions that show promise for the future.

\section{THE PHYSICS OF FAILURE AT DIFFERENT SCALES}

Now that we have established a basic layman's definition of failure, the next step is to come up with a rigorous physical explanation for how materials break. The key challenge here is that clearly not all materials are the same; glass breaks differently than a metallic paper clip, and that is different than how a muscle tear in an injury takes place. A technical definition of materials failure requires understanding different failure modes, which may be activated under a variety of different boundary conditions, and, most importantly, by the multiscale makeup of the material that controls the most fundamental unit mechanisms of failure. For all these phenomena, a consideration of physical processes at multiple time and length scales is essential in order to develop rigorous models of failure.

The most fundamental source of the difference in materials behavior lies at the atomistic scale, essentially controlled by the atomic interactions. Typically, materials feature different types of chemical bonds, which lead to significantly variant nanostructures that influence macroscale properties. In the case of glass, we observe that fracture occurs suddenly and propagates through the specimen at extremely high speeds (close to the order of sound speeds on the order of several $\mathrm{km} / \mathrm{sec}$ ). However, it is extremely tough to break a metallic paper clip by trying to pull it apart, and certainly the same type of rapid fracture as observed in glass is not found. Yet, if the material microstructure is altered by, for instance, bending a paper clip repetitively, it can eventually be broken with less effort. Muscle fibers, on the other hand, are extremely efficient in carrying loads repeatedly, but stretching them beyond their limits may lead to sudden tearing of fibers, resulting in injury. Mechanical defor- 
(a)

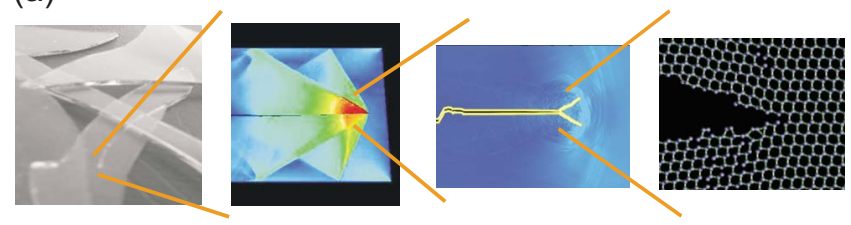

(b)

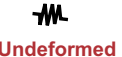

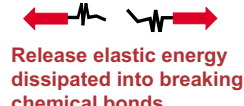

chemical bonds

FIG. 1. (Color online) Multiscale mechanisms of materials failure. (a) Multiscale view of failure of glass, from macro to nano. (b) Fracture can be envisioned as dissipation of elastic (reversible) energy. This basic view of fracture holds for a very broad range of failure phenomena, from failure of the Earth during earthquakes, failure of engineering materials, to failure of proteins in cells, tissues, or organisms (Buehler and $\mathrm{Xu}, 2010$ ).

mation of biological tissues (e.g., blood vessels) is a natural cue that initiates the formation of this very tissue through a process called angiogenesis (growth of new blood vessels) (Yung et al., 2009); however, changes in the material structure due to the buildup of calcium deposits and a heightened blood pressure might lead to catastrophic failure, causing heart attack and stroke. So what leads to these rather distinct material phenomena, and how can we formulate a fundamental physical model to predict onset of materials failure?

At a fundamental level, fracture of a material due to mechanical deformation can be understood as dissipation of elastic energy into breaking of chemical bonds and heat. This can be exemplified by envisioning an elastic material such as a rubber band; by stretching it, elastic energy is stored inside the material. At the moment of fracture, this elastic energy is dissipated, where most of the energy goes into breaking or tearing of molecules and atomic bonds and into heating up the sample. Whereas the storing of elastic energy is a process associated with the length scales of a macroscopic specimen, the tearing of molecular bonds typically happens at molecular and submolecular levels. This intimate connection of small and large is a universal hallmark of fracture, and the development of appropriate models provides the basis for exciting intellectual challenges and opportunities. Figure 1 shows the basic process of fracture, including a schematic multiscale view of failure of glass (for which crack extension via repeated breaking of interatomic bonds is a unit mechanism of fracture), as well as the mechanism of dissipation of energy during the basic unit event of fracture.

We now put the concepts discussed previously into a simple mathematical model, here done specifically for a crack in a brittle material. Cracks are one of the most prominent flaws in materials, representing either inclusions of void within materials or regions of weak adhesion. Figure 2 shows the basic energy balance during crack extension for a thin strip geometry, where a crack ranges half way through the material (also referred to as

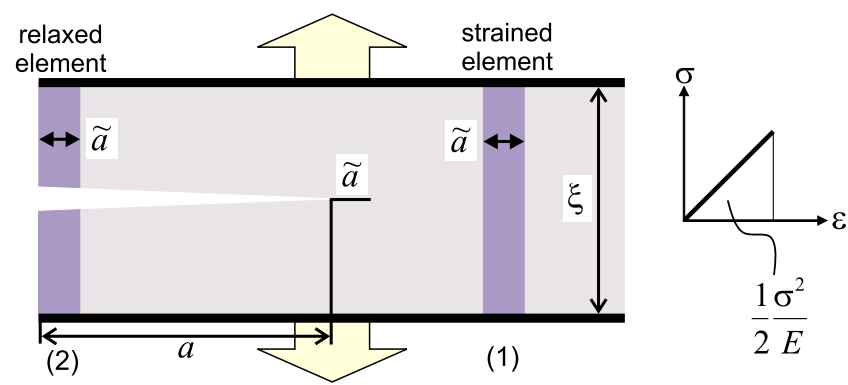

FIG. 2. (Color online) Basic energy balance during crack extension, the basic mechanism of brittle failure (e.g., of a material such as glass) in a cracked solid under remotely applied load $\sigma$. The energy stored in element (1) of width $\tilde{a}$ ahead of the crack is given by $W_{P}^{(1)}=\frac{1}{2} \xi \tilde{a} B \sigma^{2} / E$, where $B$ is the out-ofplane thickness of the specimen, $\xi$ is the width of the thin strip, $\sigma$ is the applied stress, and $E$ is the Young's modulus of the material (see right panel, where $\sigma=E \varepsilon$ ). The energy stored in element (2) is $W_{P}^{(2)}=0$ since it is completely relaxed. During crack propagation by a distance $\tilde{a}$, the energy of $W_{P}^{(1)}-W_{P}^{(2)}$ $=\frac{1}{2} \xi \tilde{a} B \sigma^{2} / E$ is dissipated. This energy is used to create two new material surfaces; thus $\frac{1}{2} \xi \tilde{a} B \sigma^{2} / E=2 \gamma \tilde{a} B$, leading to the critical fracture condition $\frac{1}{2} \xi \sigma^{2} / E=2 \gamma$, or in terms of the applied stress $\sigma=\sqrt{4 \gamma_{s} E / \xi}$.

a "semi-infinite crack"). The energy stored per unit volume in the system is equal to the area under the stressstrain curve (see right panel in Fig. 2), which for a linear elastic material is equal to $\frac{1}{2} \sigma^{2} / E$. The energy stored in element (1) of width $\tilde{a}$ ahead of the crack is given by

$$
W_{P}^{(1)}=\frac{1}{2} \frac{\sigma^{2}}{E} \xi \tilde{a} B
$$

where $B$ is the out-of-plane thickness of the specimen, $\xi$ is the width of the thin strip, $\sigma$ is the applied stress, and $E$ is the Young's modulus of the material (see right panel in Fig. 2, where $\sigma=E \varepsilon$ ). The energy stored in element (2) is

$$
W_{P}^{(2)}=0
$$

since it is completely relaxed (as no traction is applied once the atomic bonds are broken). Therefore, during crack propagation by a distance $\tilde{a}$, the energy dissipated is given as

$$
W_{P}^{(1)}-W_{P}^{(2)}=\frac{1}{2} \frac{\sigma^{2}}{E} \xi \tilde{a} B
$$

This energy is used to create new surfaces, where this is commonly measured by the surface energy $\gamma$ (the surface energy measures the energy $\Delta E$ required to create a unit area surface $\Delta A, \gamma=\Delta E / \Delta A)$. Thus, the energy balance condition is such that the change in energy given by Eq. (3) has to be equal to the total surface energy $2 \gamma \tilde{a} B$, leading to the critical fracture condition

$$
\frac{1}{2} \frac{\sigma^{2}}{E} \xi=2 \gamma
$$

Solving for the critical stress yields 


$$
\sigma=\sqrt{4 \gamma E / \xi}
$$

Equation (5) thereby provides an equation that enables us to predict the stress at which a material with a crack will begin to fail. The key issue here is to note that the basic physics behind fracture initiation is not controlled by a stress criterion, but rather by a critical energy release condition. In this spirit we can more generally define the so-called energy release rate $G$, which denotes the energy dissipated during fracture per unit of newly created fracture surface area,

$$
G=-\frac{\partial U(A)}{\partial A}
$$

where $A=\tilde{a} B$ and $U$ is the energy available for crack growth, expressed as a function of the crack surface area $A$ [where $U=-\frac{1}{2} \xi \tilde{a} B \sigma^{2} / E$ or $\left.U(A)=-\frac{1}{2} \xi A \sigma^{2} / E\right]$. Equation (6) can be rewritten as a discrete differential as

$$
G=-\frac{\Delta U(A)}{\Delta A}=\frac{1}{\left(\tilde{a}_{2}-\tilde{a}_{1}\right) B} \frac{1}{2} \frac{\sigma^{2}}{E} \xi\left(\tilde{a}_{2}-\tilde{a}_{1}\right) B=\frac{1}{2} \frac{\sigma^{2}}{E} \xi .
$$

The onset of fracture is then characterized by the condition

$$
G=2 \gamma,
$$

which resembles the condition expressed in Eq. (4). Irwin for the first time put the concept of the energy release rate outlined above into a mathematical framework that is generally applicable for a variety of geometries and loading cases (Griffith, 1921; Irwin, 1957). Thus Eq. (8) is typically referred to as the Griffith condition.

This theory describes the stability condition for cracks; once the Griffith condition is reached a small crack can propagate through the material, leading to overall catastrophic failure as the crack growths uncontrollably. This thermodynamical model can capture the link between bond breaking (expressed through the surface energy) and the overall stored energy (expressed through the energy release rate). The fracture surface energy can typically be computed from bond properties and the geometry of the crack plane with respect to the microstructure of the material, or alternatively from atomistic simulations. It is noted, however, that in many materials the creation of new fracture surfaces is not the only dissipation mechanism. For example, crack extension may be associated with amorphization at the crack tip, crack surface reconstructions, or lattice reorganization mechanisms. In these situations, the condition $G$ $=2 \gamma$ should be modified to include other dissipation mechanisms characterized by $\gamma_{\text {diss }}$, leading to $G=2 \gamma$ $+\gamma_{\text {diss }}$. Comparing this with Eq. (4) or Eq. (8) shows that the critical fracture stress increases as additional dissipation mechanisms appear, leading to

$$
\sigma=\sqrt{2\left(2 \gamma+\gamma_{\text {diss }}\right) E / \xi}
$$

Indeed, many materials engineering approaches to increase the strength of materials are based on the concept (a)

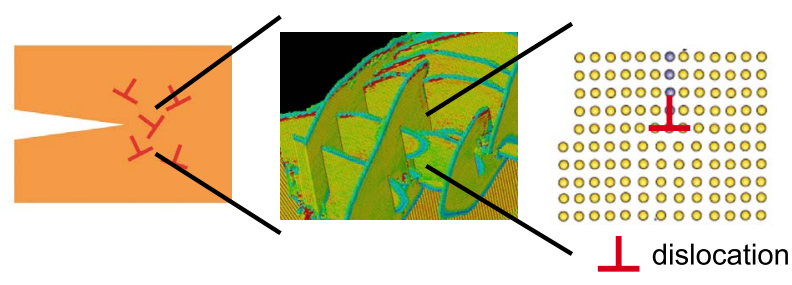

(b)

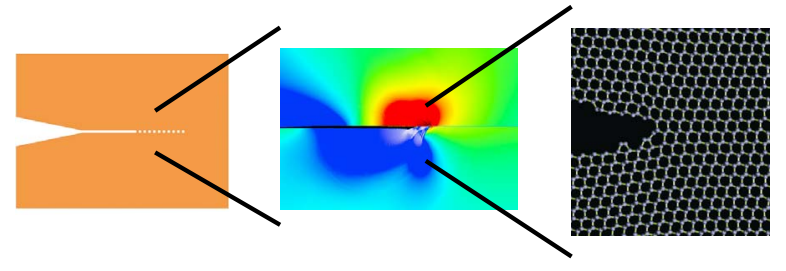

(c)

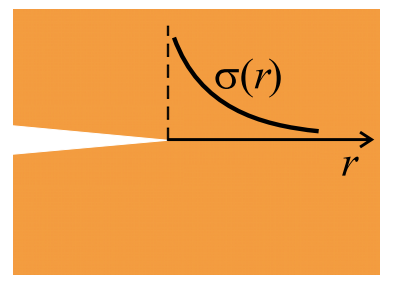

FIG. 3. (Color online) Physical mechanisms of failure in ductile (a) and brittle (b) materials, representing two fundamental failure modes. In both cases, dissipation of stored elastic energy drives the failure process. However, the mechanism of energy dissipation is different in the two cases. In (a), dislocations are the key dissipative mechanism (shown by the $\perp$ symbol). In (b) the extension of the crack through the creation of new material surfaces is the governing energy dissipation mechanism. The existence of a stress concentration around a cracklike defect shown in (c) leads to locally much higher stresses than those found further away from the crack (stress is denoted by $\sigma$, and the distance from the crack tip denoted by $r$ ). These local stresses can cause cracks to extend as the large interatomic forces induce bond breaking [(b), as in brittle materials] or bond shearing [(a), as in ductile materials] (note that the stress concentration appears at cracks or flaws in any solid, regardless if it is brittle or ductile).

of introducing additional dissipation mechanisms to prevent cracking, realized by adding small particles or alloying elements.

At a fundamental molecular scale, the most basic materials failure phenomena can be attributed to several basic atomistic mechanisms, including rupture of bonds to create new surfaces and sliding of bonds along a cleavage plane. Figure 3 shows an overview over both mechanisms. Glass, for example, has an amorphous microstructure where an orderly crystal structure is not present. In glass, failure occurs due to brittle fracture, atomic bonds break catastrophically through the material while creating new material surfaces once a critical loading condition is reached. The critical condition can be predicted by Griffith model for brittle fracture as outlined above (Griffith, 1921). In the case of a metal paper wire, for instance, one made of copper, we find an orderly face-centered cubic (fcc) crystal structure in the material. This allows reorganization of the material during failure through slipping of bonds (as opposed to 


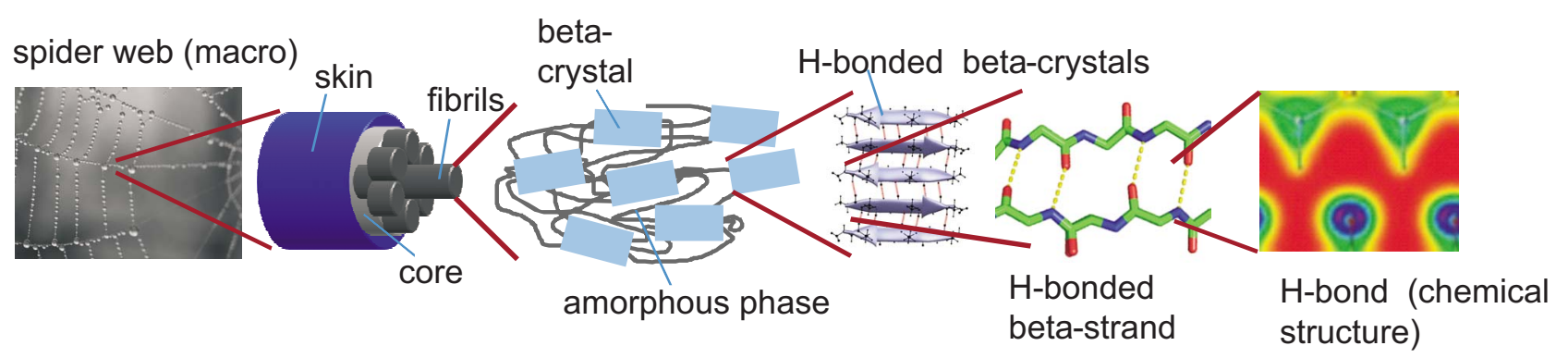

FIG. 4. (Color online) Schematic views of the hierarchical structure of spider silk and its fundamental beta-sheet protein building blocks (Keten et al., 2010). A spider web, a spider silk fiber, the microstructure of a spider silk fiber, and a detailed view of beta-sheet crystals, as well as individual H-bonded beta-strands that make up beta-sheet crystals are shown. The particular hierarchical structure of biological and natural materials makes it challenging to develop fracture models; mechanisms of failure and energy dissipation may occur at multiple scales and cannot be identified easily. Spider web image courtesy Nicolas Demars.

breaking) through crystal planes, a phenomenon known as dislocations. The reorganization of the lattice structure remains after the load is removed, leading to a permanent change of the shape of a material. This alternative microscopic deformation mode leads to what is known as ductile failure, where a large amount of energy dissipation occurs due to dislocation motion during plastic deformation, before fracture processes take place. Specifically, the emergence of dislocations increase $\gamma_{\text {diss }}$, which leads to an increased resistance of materials to catastrophic failure [see Eq. (9)]. This mediates the repeated motion of dislocations through a material, which can lead to thinning (typically referred to as necking) that eventually leads to fracture of the material. However, since these processes require more strain until they lead to catastrophic failure, ductile materials tend to be more robust with respect to the ability to tolerate large deformation.

Mechanisms can be much more diverse and complex, however, in materials with a more intricate structural makeup. In the case of biological materials such as cells, bone, or spider silk (see Fig. 4), the structural makeup of the material is extremely complex and ranges through multiple scales via the formation of hierarchical structures, involving both strong covalently bonded polypeptide chains and a myriad of weaker interactions such as salt bridges, van der Waals forces, and quite importantly hydrogen bonds ( $\mathrm{H}$ bonds). Failure of the material is yet again linked to atomistic mechanisms such as protein unfolding, molecular rupture, or sliding of biological molecules or biomolecular assemblies. Unlike other (primarily engineering) materials, our understanding of how biological materials fail under external force is limited, and the theoretical framework for understanding crossscale interactions in these materials is not yet unified. Continuum approaches empirically describe their mechanical behavior at the tissue and in some cases the cellular level, whereas biophysical theories are confined to explaining protein rupture in a case specific manner at the molecular level. As in engineering materials, the coupling between different scales is fundamentally important in understanding the mechanical properties of biological materials. Specifically, the concept of increasing the resistance to fracture by introducing additional dissipation mechanisms as shown in Eq. (9) plays an important role in understanding the material properties of biological tissues such as bone, nacre, or tendon. These materials have the capacity to dissipate much more energy than that associated with a single fracture surface or the nucleation of dislocations. Rather, numerous dissipation mechanisms are facilitated by the distinct levels of hierarchical structures found in biological materials. This type of behavior has been studied, for example, to explain the great toughness of bone (Ritchie et al., 2009) or the remarkable extensibility and flaw tolerance of protein networks in cells and tissues (Ackbarow et al., 2009).

\section{ATOMISTIC AND MOLECULAR MODELING OF MATERIALS FAILURE}

The fact that failure is directly linked to distinct atomistic mechanisms makes atomistic and molecular level modeling an indispensable tool for studying how things break. A discussion on how this is done theoretically and computationally for different types of materials is central to this Colloquium, but before proceeding further, it is worthwhile to describe the theoretical basis of molecular simulation methods. For the sake of brevity here we focus primarily on molecular dynamics simulation, a selection of force fields and a brief overview of multiscale approaches through coarse-graining techniques. Molecular dynamics (often referred to as MD) is a suitable tool for elucidating the atomistic mechanisms that control deformation and rupture of chemical bonds at nanoscale, and for relating this information to macroscopic materials failure phenomena [see, e.g., review articles and books (Vashishta et al., 1999; Rountree et al., 2002; Buehler, 2008), and recent articles from our group that describe large-scale MD simulation of brittle fracture mechanisms (Buehler, Duin, and Goddard, 2006; Buehler and Gao, 2006a, 2006b; Buehler et al., 2007)]. The objective of MD techniques is to simulate the motion of a group of atoms, generally representing the fraction of a larger system, to observe a critical phenomenon of interest, and/or to get an estimate of the global system properties. 
The basic concept behind MD is to calculate the dynamical trajectory of each atom in the material using atomic interaction potentials that describe attractive and repulsive forces in between pairs or larger groups of atoms. The interaction potentials are generally based on a mix of empirical data and first-principles based information such as quantum mechanics calculations. Solving each atom's equation of motion according to $F=m a$, positions $r_{i}(t)$, velocities $v_{i}(t)$, and accelerations $a_{i}(t)$ are calculated at each step, leading to atom trajectories that can reveal overall dynamics of the system as well as properties such as viscosity, bulk modulus, or fracture toughness. The total energy of the system is written as the sum of kinetic energy $(K)$ and potential energy $(U)$,

$$
E=K+U \text {, }
$$

where the kinetic energy is given by

$$
K=\frac{1}{2} m \sum_{j=1}^{N} v_{j}^{2} .
$$

The potential energy is a function of the atomic coordinates $r_{j}$,

$$
U=U\left(r_{j}\right),
$$

with a properly defined potential energy surface $U\left(r_{j}\right)$. The forces and accelerations are related by $a_{i}=f_{i} / m$. The forces are obtained from the potential energy surfacesometimes also called force field (or potential) —as

$$
F=m \frac{d^{2} r_{j}}{d t^{2}}=-\nabla_{r_{j}} U\left(r_{j}\right), \quad j=1, \ldots, N .
$$

The numerical problem to be solved is a system of coupled second-order nonlinear differential equations which can only be solved numerically for more than two particles, $N>2$. Typically, MD is based on updating schemes that yield new positions from the old positions, velocities, and the current accelerations of particles. For instance, in the commonly used Verlet scheme, this can be mathematically formulated as

$$
r_{i}\left(t_{0}+\Delta t\right)=-r_{i}\left(t_{0}-\Delta t\right)+2 r_{i}\left(t_{0}\right)+a_{i}\left(t_{0}\right)(\Delta t)^{2}+O\left(\Delta t^{4}\right) .
$$

The basic approach is shown in Fig. 5(a). Various fast numerical integration schemes are employed to solve the equations of motion and simulate a large ensemble of atoms representing a larger material volume; however, in particular for all-atom simulations, highfrequency vibrations of light atoms requires a time step in the order of femtoseconds $\left(1 \mathrm{fsec}=10^{-15} \mathrm{sec}\right)$ for accurate and numerically stable calculations. This limits the application of full-atomistic MD methods to nanometer size systems, at submicrosecond time scales (it is noted, however, that simulations in excess of hundreds of ns typically run for weeks or months).

The application of the MD method to long-time scale deformation and failure phenomena such as creep or fatigue, or protein folding, is particularly challenging due to the time scale issue. In recent years progress has (a)

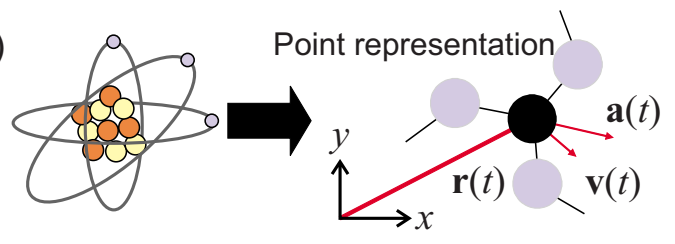

(b)
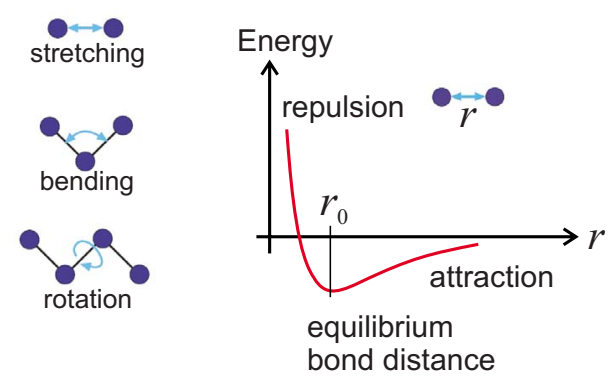

FIG. 5. (Color online) Basic approach of the molecular dynamics simulation method. (a) Atomistic structure (neutrons, electrons, and protons) replaced by a point representation in the molecular dynamics approach. (b) Illustration of the energy decomposition in classical molecular dynamics force fields, along with a representation of a simple potential function between pairs of atoms.

been made to enable atomistic and molecular level simulation of such long-time phenomena, where the methods are potentially applicable to both crystalline materials and polymers or proteins (Laioa and Parrinello, 2002; Voter et al., 2002; Kushima et al., 2009; Lau, Kushima, et al., 2009). Many of these applications are based on statistical models and include various levels of approximation for long-time scale mechanisms (Alava et al., 2006). One of the key issues of MD is that a system may be trapped in a local energy minimum, and that the escape out of the local minimum is hindered due to the lack of accessible time scales (effectively suppressing the exploration of the entire state space). However, in order to simulate certain phenomena such as protein folding, it is essential that the entire space of possible configurations can be explored. Some methods [e.g., the metadynamics approach, applied to protein modeling (Laioa and Parrinello, 2002)] overcome this limitation by enabling the system to escape out of local energy traps. Other approaches such as the replica exchange method facilitate a more extensive exploration of the state space by running copies of the system at multiple temperatures, effectively enabling us to simulate the long-term behavior of molecular assemblies. The autonomous basin climbing method is an algorithm that enables climbing out of potential minima, extending the metadynamics approach (Laioa and Parrinello, 2002) towards applications of modeling crystals and liquids and thereby facilitates the simulation of mechanisms such as creep in solids and viscosity effects in liquids at vast time scales.

Aside from limitations with respect to the system size and the accessible time scale, MD has another important limitation related to the availability of interatomic potentials for a specific material. These potential functions must be able to model the characteristic type of chemi- 
cal bonding, which can be a limiting factor for the applicability of the MD method. Materials such as silicon, iron, and steel, or colloidal systems (e.g., cement), as well as some polymers pose particular challenges with respect to the development of models for their chemical interactions and reactions. For modeling fracture this issue is particularly critical, as it involves bond breaking and bond rearrangements at the crack tip, which resembles a chemical reaction (think of bond breaking as the reverse reaction to bond formation in the synthesis of a molecule from its basic atomic constituents).

One of the strengths and a unique feature of atomistic methods is its very fundamental viewpoint of materials phenomena, a feature that is particularly important for failure processes. The only physical law that is put into the simulations is Newton's law and a definition of how atoms interact with each other. Despite this simple basis, very complex phenomena can be simulated. Unlike many continuum mechanics approaches (such as finite element methods), atomistic techniques require no a priori assumptions about the macroscale material description (e.g., elastic properties, linearity, isotropy, etc.). Once the atomic interactions are chosen according to the specific bond properties and the chemical and structural makeup of the material, the material behavior is determined, and mechanisms operating at multiple material scales are naturally captured (provided that a sufficiently large sample is simulated). Recent advances in computational power now enable the simulation of billions of particles in massively parallelized MD simulations implemented on petaflop supercomputers, reaching dimensions on the order of micrometers (Sanbonmatsu and Tung, 2007). We now proceed with an in-depth discussion of a variety of potential formulations and then discuss strategies used to bridge through even larger ranges of scales in length and time than possible by using pure atomistic models.

\section{A. Conventional and reactive force fields}

All-atom force fields are predominantly used in molecular dynamics simulations of materials at the nanoscale, as they generally are the most reliable yet computationally efficient way of studying dynamics of materials and molecules. A wide range of force fields and simulation programs are currently available, most notably embedded atom models for metals, and force fields specific to organic compounds and biomolecules such as the DREIDING, AMBER, CHARMM force fields, and programs, the OPLS force field. The GROMOS/GROMACS (Van der Spoel et al., 2005) packages are also commonly used in all-atom molecular dynamics. The NAMD (Nelson et al., 1996) program is a popular code that is capable of carrying out computations using CHARMM and other force fields. For the sake of brevity, the main aspects of the CHARMM force field will be discussed here; the basic concepts of the MD technique and force field formulations are common to all packages used in the field [for a general review, see, for instance, Ponder and Case (2003) and Mackerell (2004)].
The CHARMM force field is widely used in the protein and biophysics community, and provides a reasonable description of the behavior of proteins. The parameters in force fields are often determined from more accurate quantum chemical simulation models by using the concept of force field training (Goddard, 2006). Parameters for the CHARMM force field have been meticulously optimized and revised over the years, taking into consideration a wide variety of input including $a b$ initio results [e.g., via density functional theory (DFT)], experimental crystal structures and geometries, as well as vibrational spectra (MacKerell et al., 1998).

The CHARMM potential includes bonding and nonbonding (interaction) terms to describe short- and longrange forces between particles, where the contributions to bond stretching, bending, and rotation are individually expressed. For example, for the three contributions in the plot shown in Fig. 5(b), simple mathematical expressions are used. For bond stretching $K_{b}\left(b-b_{0}\right)^{2}$, for bond bending $K_{\theta}\left(\theta-\theta_{0}\right)^{2}$, and for bond rotations $K_{\chi}[1+\cos (n \chi-\delta)]$. In addition to these three examples, several other terms are included to model the chemical properties of proteins and nucleic acids correctly. In the CHARMM model, the mathematical formulation for the empirical energy function that contains terms for both internal and external interactions has the form

$$
\begin{aligned}
U(\vec{R})= & \sum_{\text {bonds }} K_{b}\left(b-b_{0}\right)^{2}+\sum_{\mathrm{UB}} K_{\mathrm{UB}}\left(S-S_{0}\right)^{2} \\
& +\sum_{\text {angle }} K_{\theta}\left(\theta-\theta_{0}\right)^{2}+\sum_{\text {dihedrals }} K_{\chi}[1+\cos (n \chi-\delta)] \\
& +\sum_{\text {impropers }} K_{\text {imp }}\left(\phi-\phi_{0}\right)^{2} \\
& +\sum_{\text {nonbond }} \varepsilon\left[\left(\frac{R_{\min (i, j)}}{r_{i j}}\right)^{12}-\left(\frac{R_{\min (i, j)}}{r_{i j}}\right)^{6}\right] \\
& +\frac{q_{i} q_{j}}{\varepsilon_{1} r_{i j}},
\end{aligned}
$$

where $K_{b}, K_{\mathrm{UB}}, K_{\theta}, K_{\chi}$, and $K_{i m p}$ are the bond, UreyBradley, angle, dihedral angle, and improper dihedral angle force constants, respectively; $b, S, \theta, \chi$, and $\phi$ are the bond length, Urey-Bradley 1,3-distance, bond angle, dihedral angle, and improper torsion angle, respectively, with the subscript zero representing the equilibrium positions for the individual terms.

The Coulomb and Lennard-Jones 6-12 terms constitute the external or nonbonded interactions; $\varepsilon$ is the Lennard-Jones well depth, $R_{\min (i, j)}$ is the distance at the Lennard-Jones minimum, $q_{i}$ is the partial atomic charge, $\varepsilon_{1}$ is the effective dielectric constant, and $r_{i j}$ is the distance between atoms $i$ and $j$. In the CHARMM force field, no additional terms are used for $\mathrm{H}$ bonds, since the combination of charge and Lennard-Jones contributions were verified to be adequate for describing protein, solvent, and interface hydrogen bonding. In all-atom force fields, water molecules are generally also treated explicitly. Parameters of the force field generally are specified considering a specific water model (e.g., TIP3P model for 
CHARMM) (Ponder and Case, 2003; Mackerell, 2004).

The CHARMM force field belongs to a class of models with similar descriptions of the interatomic forces; where other models include the DREIDING force field (Mayo et al., 1990), the UFF force field (Universal Force Field) (Rappe et al., 1992), or the AMBER model (Pearlman et al., 1995; Wang et al., 2001). As discussed, in CHARMM and other classical force fields, bonded terms are modeled with harmonic springs or its variations, and therefore cannot be modified (e.g., towards a different chemical state, such as from $s p^{2}$ to $s p^{3}$ ) or broken once defined by the connectivity input obtain from the topology of the molecule. Further, the atomic charges are fixed and cannot change during a simulation. These simplifications improve the simulation speed drastically and are not a major issue for most simulations studying conformational changes of proteins under ambient physiological conditions. On the other hand, simulations in extreme conditions such as mechanical perturbations (e.g., protein unfolding studies where the breaking of covalent bonds is involved) or modeling the properties under the exposure to harsh chemical environments may require reactive force fields that can take into account changes in fixed charges of the molecules, the formation and breaking of new bonds and variations in the bond order (e.g., single versus double bond) (Stuart et al., 2000; Duin et al., 2001; Brenner et al., 2002). We refer the interested reader to review articles for additional information, in particular regarding force field models (Wang et al., 2001; Mackerell, 2004; Scheraga et al., 2007; Deniz et al., 2008).

Reactive force fields represent a milestone in overcoming the limitations of classical "nonreactive" force fields, specifically their lack of the ability to describe rupture and formation of covalent bonds and their limitations in modeling chemical reactions. This is because the covalent bond terms are described using harmonic terms [see, for example, Eq. (15)], which do not provide an accurate description of the bond energetics at large bond stretch and during reformation of new bonds. For failure properties of materials (which naturally involves large bond deformation and bond rupture mechanisms), this translates into the properties of molecules at large strain, a phenomenon also referred to as hyperelasticity. These effects can have profound impact for materials failure mechanisms, as illustrated in Gao (1996) and Buehler et al. (2003) for crystalline brittle materials. Several flavors of reactive potentials have been proposed in recent years (Stuart et al., 2000; Duin et al., 2001; Brenner et al., 2002). The ReaxFF formulation of reactive potentials, originally only developed for hydrocarbons (Duin et al., 2001), have now been extended to cover a wide range of materials, including metals, semiconductors, and organic chemistry in biological systems such as proteins (Duin et al., 2003; Strachan et al., 2003, 2005; van Duin et al., 2004; Chenoweth et al., 2005; Cheung et al., 2005; Han et al., 2005; Nielson et al., 2005; Buehler, Duin, and Goddard, 2006; Buehler, 2007b; Buehler et al., 2007). To describe the details of bond stretching and breaking, a bond length-bond order relationship is em- ployed to obtain smooth transition from nonbonded to single, double, and triple bonded systems. All connectivity-dependent interactions (that means valence and torsion angles) are formulated to be bond-order dependent. This ensures that their energy contributions disappear upon bond dissociation so that no energy discontinuities appear during reactions. Similar to the CHARMM model, the reactive potential also features nonbonded interactions (shielded van der Waals and shielded Coulomb). The reactive formulation uses a geometry-dependent charge calculation scheme that accounts for polarization effects and modeling of charge flow, assigning a partial charge to each atom at each integration step and thereby includes important quantum mechanical details about interatomic bonding. Due to the increased complexity, reactive potentials can be about 20-30 times more expensive than conventional models. A comprehensive review of reactive force fields for modeling failure is beyond the scope of this Colloquium, partly because different materials require significantly unique modeling methods and potentials. In the following we present two examples that illustrate the significance of using reactive force fields in describing the failure of a crystal of silicon and a protein molecule.

Figure 6 shows how a reactive force field has been applied to describe fracture of silicon under tensile load [loading condition, see Fig. 6(a)], where a more accurate description of the details of chemical bonding has proven to be crucial to match simulations of silicon fracture with experiment (Buehler, Duin, and Goddard, 2006; Buehler et al., 2007). In the example shown in Fig. 6 , a hybrid multiparadigm technique was used where the computationally expensive ReaxFF model was only used in a small region surrounding the crack tip, while the rest of the domain was described using a computationally less expensive Tersoff potential. The advantage of this algorithm is that it dynamically identifies regions in the simulation domain that undergo large deformation, where the ReaxFF description is mandatory in order to provide an accurate representation of the changes of the bonding characteristics under large stretch. Furthermore, the comparison between a pure Tersoff model and the hybrid ReaxFF-Tersoff model shown in Fig. 6(b) illustrates the significance of providing an accurate representation of chemical bond breaking events for modeling fracture. The failure of the Tersoff potential to accurately model the details of bond breaking under large stretch explains why the crack does not extend in this case, in contrast with experimental results. Including a fully reactive full chemistry description through the use of ReaxFF close to the crack tip (where bond breaking occurs) provides an accurate representation of crack dynamics, in agreement with experimental studies [for further details see Buehler, Duin, and Goddard (2006) and Buehler et al. (2007)].

Figure 7 shows the results of stretching and breaking a small protein molecule with covalent cross links under tensile loading (Buehler, 2007b). The study shown here reveals the differences between a nonreactive (CHARMM) force field and the ReaxFF reactive force 
(a)

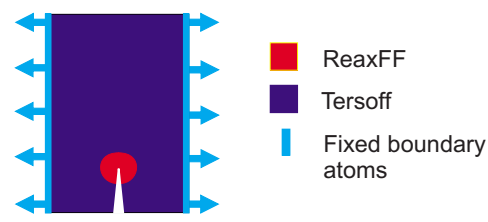

(b)

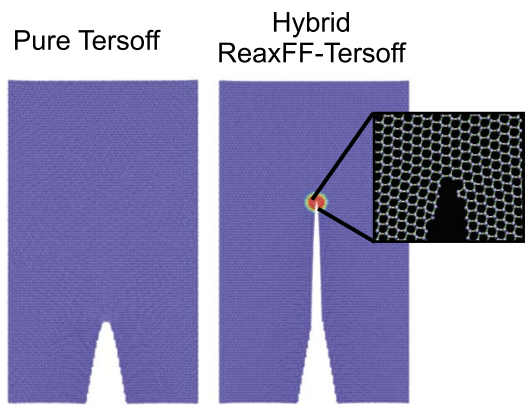

FIG. 6. (Color online) Multiparadigm molecular dynamics simulation of dynamic fracture of silicon (Buehler, Dodson, Meulbroek, Duin, and Goddard, 2006; Buehler, Duin, and Goddard, 2006; Buehler et al., 2007), carried out based on a pure Tersoff model and a hybrid ReaxFF-Tersoff model. The hybrid model schematically shown in (a) describes the fracture mechanics of silicon by a combination of a simple Tersoff force field in regions far away from bond rupture events, with the ReaxFF reactive force field used to more accurately describe the rupture processes at the crack tip. (b) Comparison between (left) the pure Tersoff model and (right) the hybrid ReaxFFTersoff model. In the pure Tersoff model the crack does not extend, in contrast with experimental results. In the hybrid model where an accurate representation of the chemistry of bond breaking is provided at the crack tip, the crack extends under application of load. This comparison illustrates the significance of providing an accurate representation of chemical bond breaking events for modeling fracture.

field. Figure 7(a) shows snapshots as the molecule is being stretched, modeled using the reactive model. As the molecule is being pulled, the covalent cross-links (disulfide bonds) within the molecule break. These breaking points correspond to the peaks in the force-extension plot shown in Fig. 7(c), and the force drops significantly after each breaking point as the elastic energy stored in the protein is released. In the case of the CHARMM model [Fig. 7(b)], bond breaking cannot be described, and the force continues to rise once the covalent crosslink within the protein is being stretched [see Fig. 7(c)]. The results shown here clearly illustrate the significance of a reactive force field approach in modeling the failure of molecules, specifically when the breaking of covalent bonds is involved.

\section{B. Multiscale simulation techniques}

Albeit providing a rather accurate description of macromolecules, all-atom modeling approaches have historically been prohibitively extensive when large systems and long simulation times must be considered. This led to the development of coarse-grained models (Tozzini, 2005), which provide a simplified representation of mac- (a)

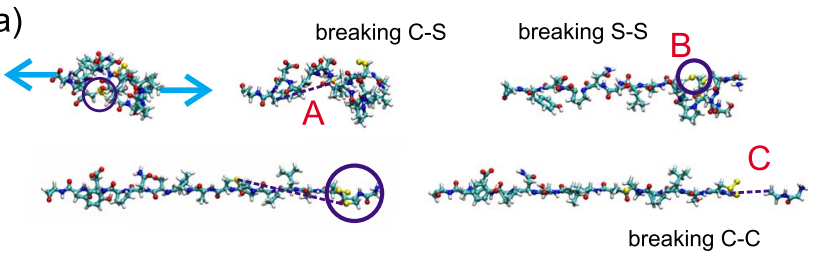

(b)

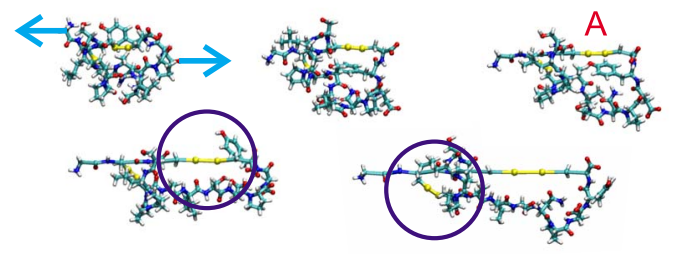

(c)

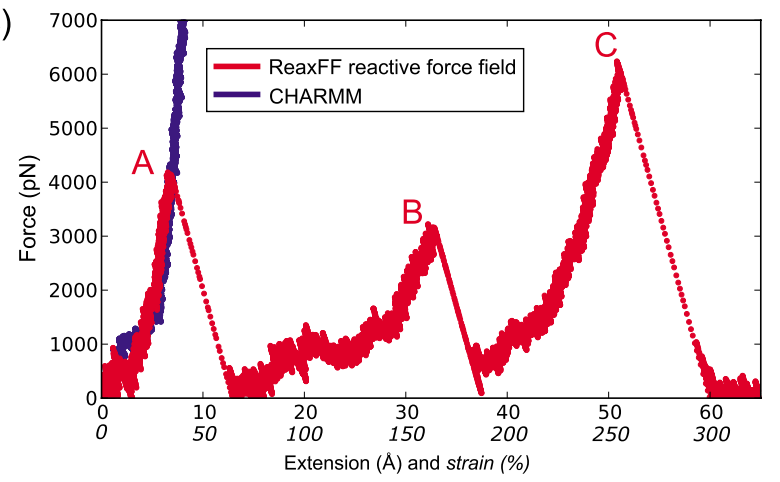

FIG. 7. (Color online) Breaking a single protein molecule by pulling at the ends of a small protein $(\alpha$ conotoxin PnIB from conus pennaceus; PDB identification code $1 \mathrm{AKG}$ ) (Buehler, 2007b). The study shown here reveals the differences between a nonreactive (CHARMM) force field and the ReaxFF reactive force field. (a) Snapshots as the molecule is being stretched, modeled using the reactive model (ReaxFF). As the molecule is being pulled, the covalent cross-links (disulfide bonds) within the molecule break. These breaking points correspond to the peaks in the force-extension plot shown in (c); and the force drops significantly after each breaking point as the elastic energy stored in the protein is released. In the case of the CHARMM model (b), bond breaking cannot be described, and the force continues to rise once the covalent cross-link within the protein is being stretched (c).

romolecules employing less degrees of freedom and simple bonded and nonbonded interactions that can be more rapidly calculated in each time step (see Fig. 8). The integrated use of simulation methods with different computational expense and accuracy is referred to as multiscale modeling, where a systematic link is established between multiple scales. The concept is shown in Fig. 9, including the representation of handshaking between different methods to pass information systematically from lower levels to coarser, larger scales. The figure also plots relevant experimental techniques that overlap with corresponding computational techniques.

Coarse-grained models have so far been successfully applied to a wide range of problems including protein folding, allostery, aggregation, and molecular biomechanics, and multiscale description of complex materials such as bone. The various approaches used in particular 


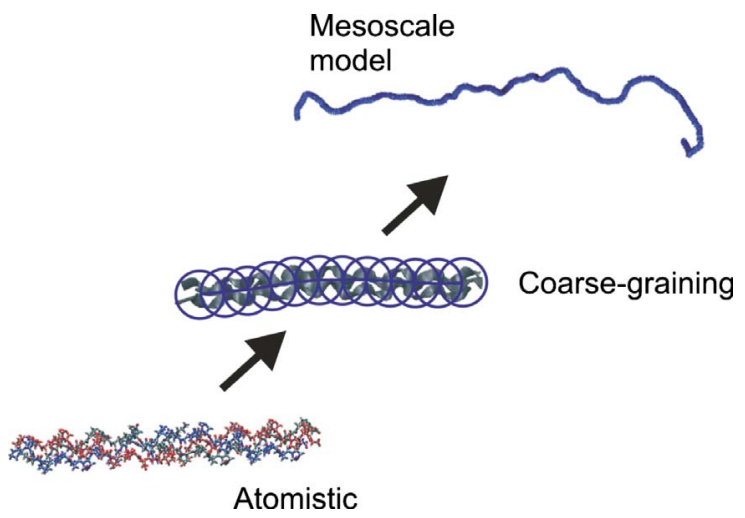

FIG. 8. (Color online) Illustration of coarse-graining approach for a simple one-dimensional fibrillar protein filament (collagen). This schematic shows how a full atomistic representation is coarse-grained and used in a mesoscale model formulation. This mesoscale model formulation enables one to reach much larger time and length scales. The systematic parametrization from the bottom up provides a rigorous link between the chemical structure of proteins (for example, through their amino acid sequence) and the overall functional material properties. This computational approach is a key component in the advancement of materiomics as it provides us with the ability to reach microsecond and micrometer length scales.

for biological materials are reviewed here. Single-bead models are perhaps the earliest approach taken for studying macromolecules. The term single bead derives from the idea of using single beads (masses) for describing each amino acid in a protein structure. The elastic network model (ENM) (Tirion, 1996), Gaussian network model (Haliloglu et al., 1997), and the Go-like model (Hayward and Go, 1995) are well-known examples of this simplistic approach. Simple models such as ENM and Go-like models treat each amino acid as a single bead located at the $\mathrm{C}_{\alpha}$ position with mass equal to the mass of the amino acid. The beads are interconnected by harmonic or nonlinear springs representing the covalently bonded protein backbone. In the Go-like models, an additional Lennard-Jones term is included in the potential to describe short-range nonbonded native interactions between atoms within a cutoff distance. Despite their simplicity, these models have been extremely successful in explaining thermal fluctuations of proteins (Tozzini, 2005) and have also been implemented to model the unfolding problem to elucidate atomic-level details of deformation and rupture that complement experimental results (West et al., 2006; Sulkowska and Cieplak, 2007; Dietz and Rief, 2008). A more recent direction is coupling of ENM models with a finite elementtype framework for mechanistic studies of protein structures and assemblies (Bathe, 2008). Due their simplicity, single-bead models have several shortcomings. With classic ENM, only harmonic deviations from the initial configuration are possible. In the Go model, native interaction definitions lead to a minimally frustrated landscape which is highly biased towards the input configuration of the molecule. Such models therefore cannot predict folding or unfolding intermediates and meta-

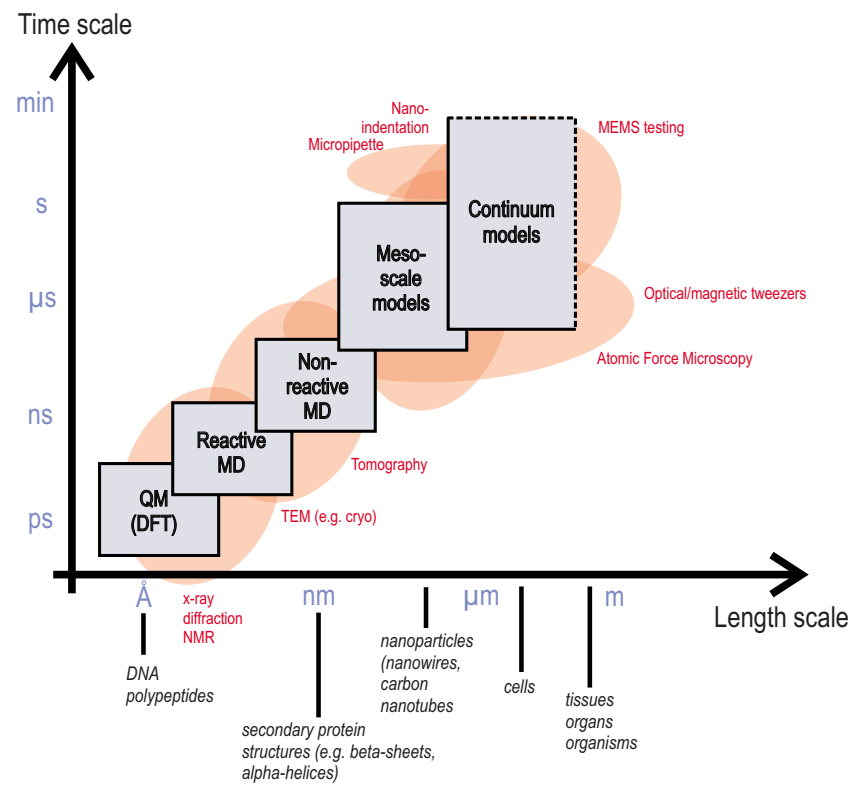

FIG. 9. (Color online) Experimental, theoretical, and computational tools for the characterization and modeling of deformation and failure of materials, plotted over their respective time and length scale domain of applicability. Experimental methods include x-ray diffraction, TEM (transmission electron microscopy), AFM (atomic force microscopy), OT/MT (optical/magnetic tweezers), and MEMS (mechano-electromechanical system) testing, as well as nanoindentation. Frequently used theoretical and simulation tools include quantum mechanics (DFT), molecular dynamics, coarse-grained models, mesoscale atomistically informed continuum theories, and continuum models. The lower part indicates respective classes and scales of materials that can be studied with these types of techniques. Adapted from Buehler and Yung, 2009.

stable states. The explicit treatment of protein-solvent interactions, non-native interactions and $\mathrm{H}$ bonds is also not possible with single-bead models. It is now widely accepted that for protein unfolding studies, the results obtained using such models are only qualitative at best, although they may reveal important aspects of topologydependent mechanical resistance (West et al., 2006; Sulkowska and Cieplak, 2007; Dietz and Rief, 2008), and can thus be used to improve our understanding of structure-property links.

Using more than one bead per amino acid can lead to a more detailed description of macromolecules. In the simplest case, the addition of another bead can be used to describe specific side-chain interactions (Bahar and Jernigan, 1997; Marrink et al., 2007; Monticelli et al., 2008). Four to six bead models capture even higher amount of detail by explicit or united atom description for backbone carbon atoms, side chains, carboxyl, and amino groups of amino acids. A successful implementation of this approach is the coarse-grained models developed for studying folding and aggregation in proteins using discontinuous molecular dynamics (Nguyen and Hall, 2004, 2006). Although multibead models have superior qualities compared to single-bead descriptions, dozens of additional energetic terms involving pseudo- 
bonds and other means to avoid complex dihedral or improper potentials that stabilize the conformation of the polypeptide chain have to be introduced for generic models. Even with the introduction of these terms, some of which are physically not intuitive, the models offer limited applicability, as the defined side chain interactions are only valid for simple residues such as glycine and alanine. More complex yet computationally efficient potentials that intrinsically take into account sequence specificity are extremely challenging to develop, thus making readily available all-atom descriptions and simulation packages more favorable for most applications. Practical methods of developing coarse-grained models that have wide applicability remain challenging for polymers and proteins in particular.

More recently, coarser-level modeling approaches have been applied to model biomolecular systems at larger time and length scales. These models typically employ superatom descriptions that treat clusters of amino acids as "beads," as shown schematically in Fig. 8 (for the case of tropocollagen molecules). In such models, the elasticity of the polypeptide chain is captured by simple harmonic or anharmonic (nonlinear) bond and angle terms. These methods are computationally quite efficient and capture shape-dependent mechanical phenomena in large biomolecular structures (Arkhipov et al., 2006), and can also be applied to collagen fibrils in connective tissue (Buehler, 2006) as well as mineralized composites such as nascent bone (Buehler, 2007a). Coarse-grained techniques based on results from QM or all-atom MD modeling approaches show great promise as they can run much faster than multiatom descriptions for molecular building blocks such as proteins and are relatively easier to implement than multibead potentials that require complicated energy terms for achieving the correct molecular geometry. Since such high-level precision is not sought after in these coarser methods, simpler terms are generally used to achieve the global structure and dynamical information of the system.

In addition to methods that uniformly simplify a complex system by coarse graining, hybrid approaches that employ atomistic details at active regions of biomolecular systems or crystalline solids as shown in Fig. 6 in the silicon fracture example also show great promise (Neri et $a l ., 2005)$. The use of hybrid approaches in protein modeling has been pioneered early on through the use of so-called QM-MM methods (where quantum mechanical descriptions is used at enzymatic sites, and nonreactive models are used elsewhere in the protein). Overall, one can identify two fundamental viewpoints in multiscale modeling: (1) Employing different levels of detail or fidelity in a single model (hybrid or concurrent approaches), and (2) enabling scale transitions by extraction or passing of key information (i.e., parameters) to higher scales based on more accurate simulations at smaller scales (hierarchical approaches). Developing accurate models that can predict not only the overall structural behavior but also processes such as bond formation, self-assembly as well as molecular and macroscale failure of materials is an area of great interest for phys- ics, materials science, and medical applications. Which of the two approaches (i.e., hybrid or hierarchical approach) is better suited depends strongly on the type of application and the specific properties that are simulated.

\section{CASE STUDIES: FAILURE OF MATERIALS, FROM NANO TO MACRO}

In this section we present a review of failure mechanisms of three classes of systems, starting with earthquakes (failure of the Earth's crust), focusing on bone, and finally discussing failure of molecules. We highlight commonalities between all three examples discussed here.

Bridging the gap between vastly different scales in a single model remains a challenge as cross-scale interactions and hand shaking between regions of different detail demand a rigorous theoretical basis and access to large-scale computational resources. For instance, incorporating molecular-level detail in simulations of earthquakes would be extremely challenging in this regard; however, studies of bone and single molecules based on bottom-up modeling approaches have become increasingly popular. Similar simulations can be carried out for macroscale systems using information passing across scales, for instance, by employing large representative particles (superatoms) as in the case of a coarse-grained model.

\section{A. Failure of the Earth's crust: Earthquakes}

In earthquakes, the sudden rupture and slipping of the tectonic plates affects the ground's ability to provide stable foundation for the built environment. Elastic waves emitted by these rupture processes may lead to strong vibrations of the ground, inducing great damage in bridges, buildings, and roads. Figure 10 shows the basic fracture process associated with an earthquake. Two tectonic plates are slowly sheared against each other over the course of many years (corresponding to remotely applied loading), while elastic energy is stored in the system (through deformation of the crust). The elastic energy is suddenly released in a catastrophic event once the earthquake occurs as the two tectonic plates slides against each other, while energy is dissipated in frictional processes, which is characterized by propagation of a cracklike rupture front. This process of energy storage and subsequent dissipation reflects the mechanism shown in Fig. 1(b).

Figure 11 shows the geometry of an earthquake in Kocaeli, Turkey, as it occurred in 1999 (Sekiguchi and Iwata, 2002). The plot shows the path of the earthquake along a weak plane in the Earth's crust. This earthquake spread almost linearly from the initiation point in Kocaeli towards Eften Lake and Izmit Bay. Based on geophysical measurements, geologists have also analyzed the dynamics of this rupture event, in order to identify the speed at which earthquakes occur. Figure 12 shows the analysis of the position over time (and thereby pro- 


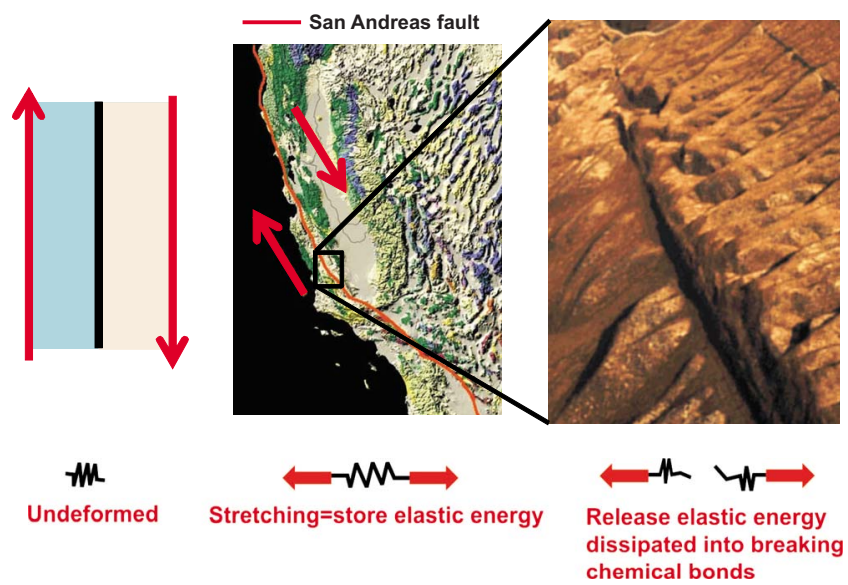

FIG. 10. (Color online) Basic fracture process associated with an earthquake, here exemplified for the case of the San Andreas fault. Two tectonic plates are sheared against each other, while elastic energy is stored in the system, which is released in a catastrophic event once the earthquake occurs. Energy dissipation mechanisms include friction between along the fault line, where local slip occurs. Maps and photographs of the San Andreas fault courtesy of the U.S. Geological Survey (the detailed aerial view of the San Andreas fault is taken near Carrizo Plain, Central California).

viding immediate insight into the speed) of this earthquake. The analysis shows that that the rupture propagates intersonically after a short initial phase of subsonic growth, with speeds in excess of several $\mathrm{km} / \mathrm{sec}$. The understanding of dynamics of earthquakes is important to develop better models to predict future events; thereby, a precise knowledge of the speed of propagation and the path is crucial to estimate the resulting damage in infrastructure. For example, the occurrence of intersonic speeds results in shock fronts, with very strong and sudden displacements of the crust. These types of mechanisms can lead to rather severe structural damage in natural and built infrastructure. Intersonic propagation of earthquakes was first observed in this Kocaeli earth-

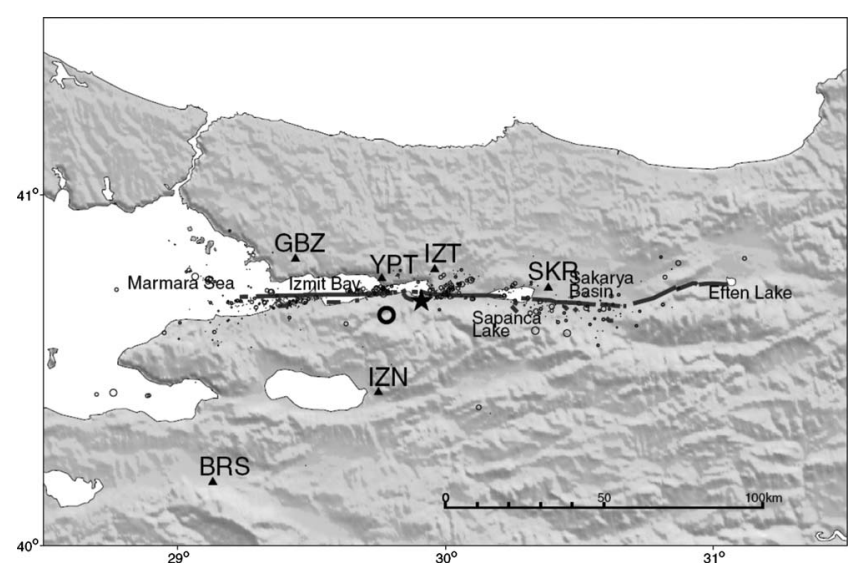

FIG. 11. Earthquake dynamics of the 1999 Kocaeli, Turkey earthquake (Sekiguchi and Iwata, 2002). The plot shows the path of the earthquake along a weak plane in the Earth's crust. From Sekiguchi and Iwata, 2002.

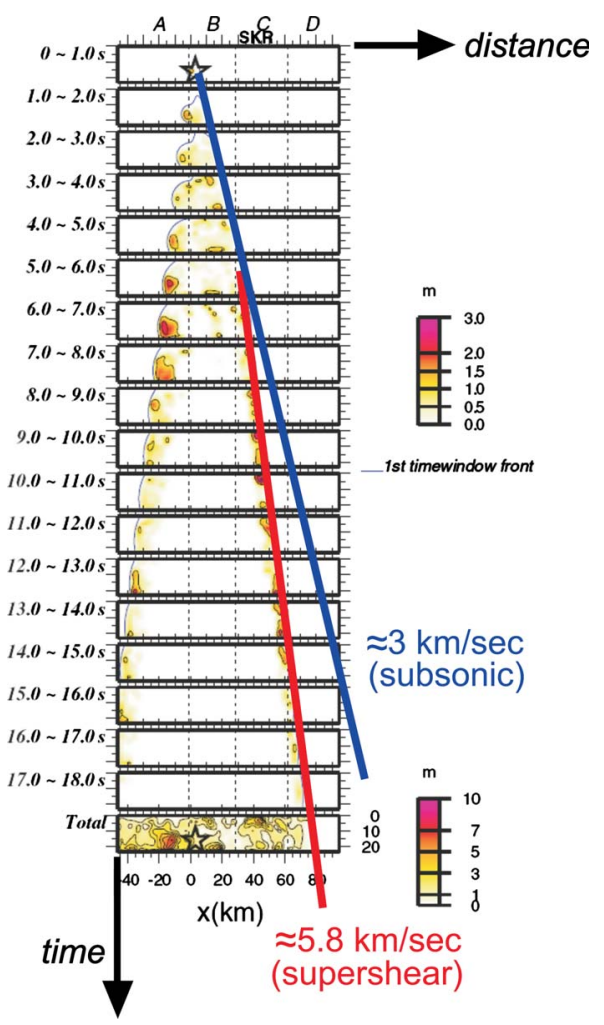

FIG. 12. (Color online) Analysis of the dynamics (and speed) of the earthquake's rupture front. The location (distance) of the front of the earthquake is shown as a function of time. It propagates at a speed of approximately $3 \mathrm{~km} / \mathrm{sec}$ initially (subsonic), then followed by a sudden jump to a much higher propagation velocities of around $5.8 \mathrm{~km} / \mathrm{sec}$. This propagation is faster than the speed of shear wave (thus referred to as "supershear" or "intersonic"). From Sekiguchi and Iwata, 2002.

quake, even though the possibility for the existence of this phenomenon has been proposed earlier based on theoretical studies (Burridge, 1973; Andrews, 1976; Freund, 1990).

The phenomenon of intersonic rupture propagation has been investigated further since the initial observation in Turkey. In particular, experimental testing of socalled "laboratory earthquakes" put forth by a group at Caltech provide an excellent approach in further identifying underlying features and mechanisms (Rosakis et al., 1999; Rosakis, 2002; Xia et al., 2004, 2005; Rosakis et al., 2006). In these experiments (setup shown in Fig. 13), the Earth's crust is scaled down and modeled by a polymer slab, and the existence of tectonic faults is modeled using a weak plane in the polymer. Under application of shear and/or tensile or compressive loading, researchers have been able to identify important underlying mechanisms and dynamical events in this setting, such as the understanding the transition from subsonic to intersonic rupture propagation. Figure 13 confirms that laboratory earthquakes reveal a similar phenomenon leading to rupture propagation faster than the shear wave speed, as can be seen in Fig. 13(c) through the existence of shock fronts. Among other contributions, these experiments 
(a)

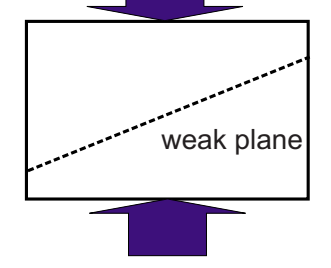

(b)

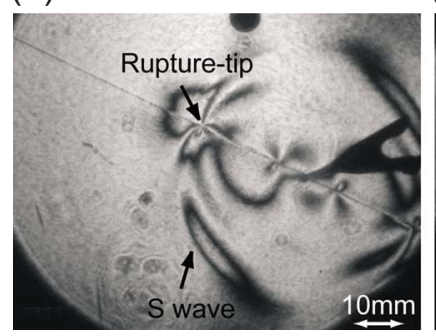

(c)

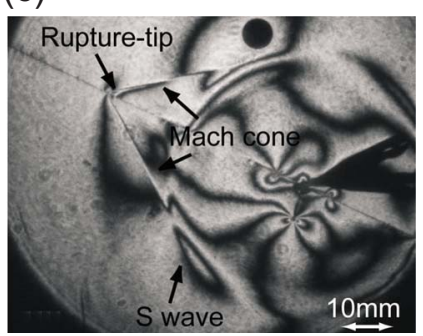

FIG. 13. (Color online) Laboratory earthquakes reveal a similar phenomenon of intersonic rupture along a shear fault, mimicking the phenomena observed in earthquakes. The Mach cones in (c) show intersonic crack propagation, similar as observed in earthquakes (see Fig. 11). From Xia et al., 2004.

suggested that intersonic fracture is possible through socalled mother-daughter mechanism, where a secondary crack is born ahead of the primary (mother) crack. The study of fundamental rupture mechanism has also been extended down to atomic scales (Holland and Marder, 1999; Gao et al., 2001; Gerde and Marder, 2001; Buehler et al., 2003; Buehler and Gao, 2006b). Figure 14 shows results of a molecular dynamics simulation of interfacial rupture along a weak layer between two elastically dissimilar materials (Buehler, 2008). This represents a similar phenomenon of a rupture propagating faster than the speed of shear waves, also enabled through a motherdaughter mechanism similar as in experimental studies of interfacial cracks. The intersonic crack speed is visible in the shock fronts that can be seen in the visualization.

These examples show that some basic features of dynamics of ruptures (specifically, the mother-daughter crack mechanisms and the existence of supershear cracks) can be observed at multiple scales-at the scale of tens of kilometers, on the order of tens of centimeters, and at the atomic scale. It is noted, however, that even though the phenomena of failure at different levels show similar features, the details of the failure processes can be quite different (compare, for example the chemical bond breaking mechanisms in a single crystal at nanoscale with rupture mechanisms of earthquakes at the scales of centimeters to meters that occur in the shear zone).

\section{B. Failure of bone: Fracture processes in injury}

After the discussion of failure at the scale of the Earth (on scales of hundreds to thousands of kilometers), we now proceed with a discussion of failure in the context of biological systems. Biological protein materials fea- (a)

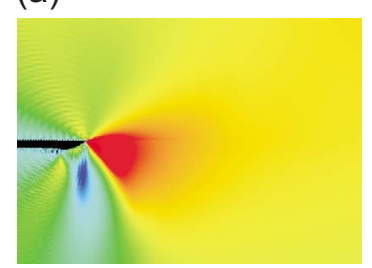

(c)

(b)

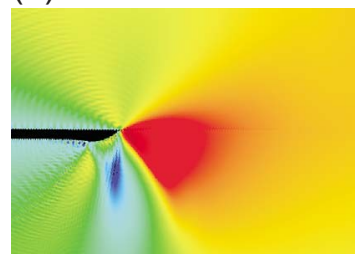

(d)

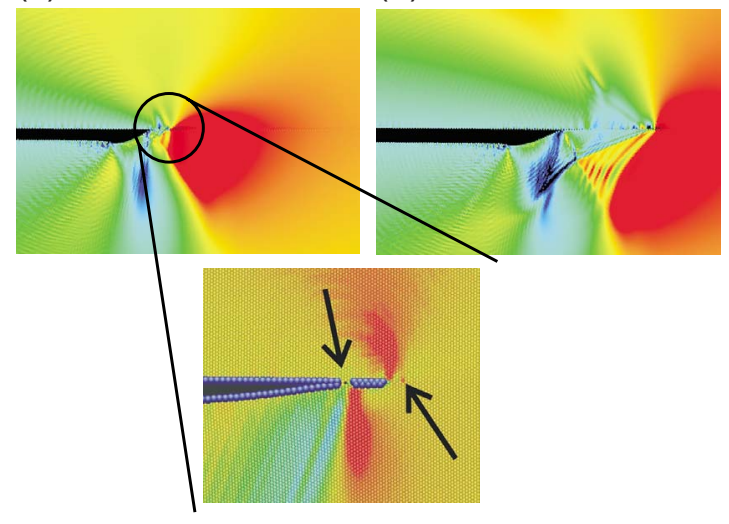

FIG. 14. (Color online) Molecular dynamics simulations of interfacial failure between two dissimilar materials (Buehler, 2008). A rupture propagates along a weak plane between two elastically dissimilar materials, moving at an intersonic speed [see shock fronts in (d)]. Intersonic fracture is possible through so-called mother-daughter mechanism where a secondary crack is born ahead of the primary (mother) crack. This mechanism is illustrated in the blowup in (c) (the two arrows indicate the mother and daughter crack, respectively).

ture hierarchical structural components to constitute a diverse range of functional physiologic materials. The analysis of mechanical properties of protein materials is an emerging field that utilizes mechanistic insight, based on structure-process-property relations in its biological context, to probe deformation and failure phenomena at the molecular and microscopic level. Thereby, the study of materials failure is a particularly important aspect in the context of injuries and disease (Buehler and Yung, 2009). Proteins constitute critical building blocks of life, forming a diverse group of biological materials, ranging from spider silk to bone, tendon to the skin, all which play an important role in providing key functions to biological systems (Lakes, 1993; Weiner and Wagner, 1998; Alberts et al., 2002; Wang and Stamenovic, 2002; Aizenberg et al., 2005; Fratzl and Weinkamer, 2007; Taylor et al., 2007; Buehler and Yung, 2009). Protein molecules form the basic constituents of this group of biological protein materials. These materials are distinct from the conventional references to structure and material, as it connotes the merger of these two concepts through hierarchical formation of structural elements that range from the nanoscale to macroscale. Protein materials are abundant in biology and play a crucial role in the biological function of all cells and tissues within organisms. Many such materials with mechanical function form structural filaments, trusses, or fibers while others retain 
(a)

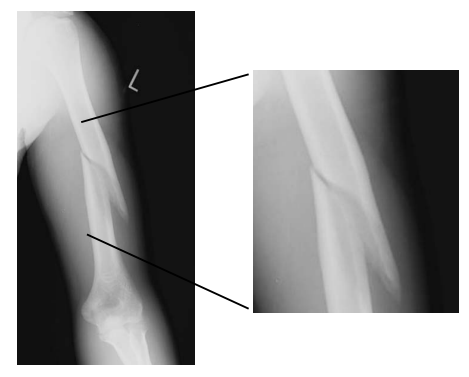

(b)

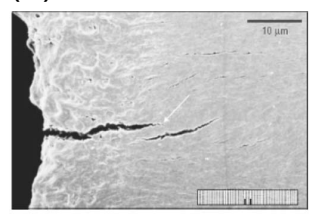

FIG. 15. Example of fracture of bone, a common form of body injury. (a) A macroscopic view of bone failure. (b) SEM micrographs of bone fracture experiments as reported by Nalla et al. (2003). From Nalla et al., 2003.

the globular structure of their protein constituents. The cascaded arrangements of building blocks at defined length scales form hierarchical structures (e.g., molecules, filaments, and mesoscale structures) that control a material's properties, including their propensity towards failure.

One of the most intriguing protein materials found in nature is bone, a biological material composed out of assemblies of tropocollagen molecules and tiny hydroxyapatite mineral crystals, forming an extremely tough, yet lightweight, adaptive and multifunctional material. Here we focus in particular on the process of bone fracture, a common type of body injury as shown in Fig. 15 (this illustration shows both a macroscopic and microscopic view of bone failure). Bone has evolved to provide structural support to organisms, and therefore, its mechanical properties are of great physiological relevance. In this section, we review the structure and properties of bone, focusing on mechanical deformation and fracture behavior from the perspective of the multidimensional hierarchical nature of its structure. The hierarchical structure of bone is shown in Fig. 16. Bone is a hierarchical composite material composed out of assemblies of tropocollagen molecules, mineral crystals, as well as water and ions and other biopolymers.

Bone derives its resistance to fracture with a multitude of deformation and toughening mechanisms at many of these size scales, ranging from the nanoscale structure of its protein molecules to its macroscopic physiological scale. Recall that the initiation of failure of a material is given by the Griffith condition [see Eq. (9)], $G=2 \gamma+\gamma_{\text {diss }}$ and shown in Fig. 2. The extreme resistance of bone against failure is due to the hierarchical structure of bone, which provides the basis for multiple energy dissipation mechanisms, each of them concurrently operating at their own level and enabling rather significant energy dissipation levels much higher than in conventional single hierarchy materials. Overall, this leads to an effective increase of $\gamma_{\text {diss }}$, and thus prevents cracks from easily spreading in bone. Specific examples of nanoscopic energy dissipation mechanisms include the uncoiling of molecules (here, the deformation of tropocollagen molecules), the sliding of molecules and molecular arrays against each other, and the breaking of

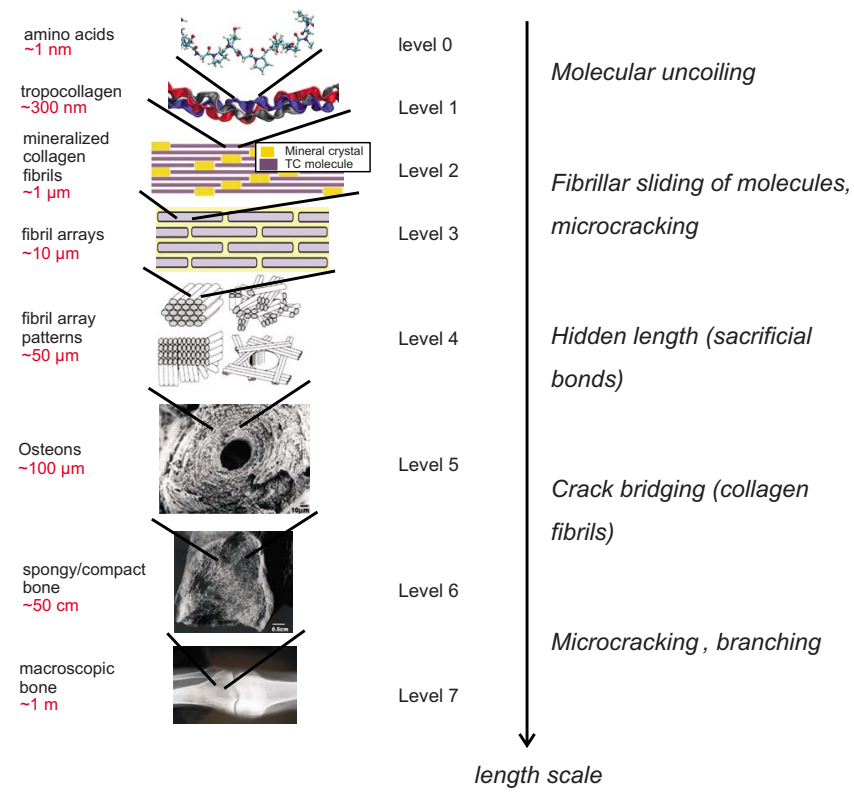

FIG. 16. (Color online) Hierarchical structure of bone, showing seven hierarchies, and their relation to the mechanisms of mechanical properties (bottom three inlays from Weiner and Wagner, 1998; figure adapted from Ritchie et al., 2009). Collagen protein molecules ("tropocollagen"), formed by three chains of amino acids, provide the structural basis mineralized collagen fibrils, the smallest building block of bone. Several mineralized collagen fibrils form fibril arrays, linked by an organic phase. Several fibril arrays (or collagen fibers) form geometric fibril array patterns, which provide structure to cellular components of bone; this is also known as the lamellar structure of bone. The boundaries between the packets of fibers comprise the lamellar interfaces. This microstructure of bone forms distinct mesoscale structural arrangements, such as spongy/compact bone and eventually macroscopic bone. Distinct toughening mechanisms occur at each level of hierarchy. Molecular uncoiling and intermolecular sliding of molecules are observed at the smallest level of tropocollagen molecules and mineralized collagen fibrils. Microcracking and fibrillar sliding are observed at the level of fibril arrays. At larger levels, the breaking of sacrificial bonds contributes to increasing the energy dissipation capacity of bone at the interface of fibril arrays. Crack bridging by collagen fibrils is observed at scales of several hundred micrometers, as well as microcracking and crack branching at scales of centimeters and beyond. Cortical (compact) bone is the dense bone that is found at the surface of all bones; trabecular (cancellous) bone is spongy with struts of order 100-200 $\mu \mathrm{m}$ in diameter and holes of approximately $1 \mathrm{~mm}$ in diameter. Notice the similarity of the structural makeup in multiple hierarchies to the structure of spider silk shown in Fig. 4.

so-called hidden sacrificial bonds. At microscopic and macroscopic scales, mechanisms such as crack bridging (resistance to crack extension due to presence of collagen fibrils orthogonal to the crack extension) and microcracking or branching contribute to an increase in bone's toughness (Ritchie et al., 2009). This variety of mechanisms does not only increase bone's overall resistance to fracture. Moreover, it increases the robustness of bone to fail, since its toughness is retained at rela- 
tively large values even if some of these mechanisms cease to operate (Nalla et al., 2003, 2005, 2006; Koester et al., 2008; Ritchie et al., 2009).

Another important issue in understanding bone's resistance to failure is its intrinsic ability to remodel, that is, to repair itself to remove damage or to adapt to new operational boundary conditions (Taylor et al., 2007). Bone can repair cracks by adding new tissue, and it can change its shape to adapt to new loading conditions by removing tissue and adding it at other locations [for an extensive review of this issue, see Taylor et al. (2007)]. This ability of bone to dynamically adapt to changes in loading conditions remains a major frontier in bone materials science. Currently, few physics-based models exist that present physically sound models for these complex phenomena. Concepts such as self-similarity and selforganized criticality may provide insight into the complexity of fracture mechanisms in a variety of biological materials, including bone, connective tissue, and perhaps even failure of organs and organisms (Bak et al., 1988; Garcimartin et al., 1997).

Despite all these mechanisms that help bone to become more resistant against failure, bone can indeed break-if the applied forces are too large, as it frequently occurs in sports injuries or accidents. It remains a major issue to identify appropriate techniques to measure bone's structural features and from that identify its likelihood for failure. Such diagnosis tools would be particularly useful for elderly patients, for whom bone fracture remains a cause for immobility and a trigger for other diseases. Recent advances in developing novel diagnostic tools could enable us to predict the risk for bone fracture based on a relatively simple mechanical testing procedure, as illustrated in the Reference Point Indentation instrument (Diez-Perez et al., 2010). Structural changes in bone as patients age can lead to a significantly enhanced likelihood for bone to break.

\section{Failure at molecular level: $\mathbf{H}$-bond rupture in protein materials}

At a fundamental level, material failure is linked to how atoms and molecules detach from each other through nanoscale processes. For instance, in order to understand the failure mechanics of proteins and biomolecular assemblies from a physical science perspective, we need to identify the universal and diverse features of polypeptides at atomic resolution and the relative importance of these features on mechanical properties of these materials. This calls for a simplified framework that is focused on the general rather than the specific properties of proteins. Here we review an approach that was recently proposed to describe failure of protein materials at the molecular level as a case study for modeling failure in nanoscale materials.

The ultrastructure of protein materials such as spider silk, muscle tissue, or amyloid fibers consists of betasheet structures that form rather strong $\mathrm{H}$ bond assemblies (Fig. 4). These materials are unique in their making as they employ not only covalently bonded polypeptide chains but also $\mathrm{H}$ bonds that give rise to unique folds and nanostructural arrangements of proteins by forming intramolecular as well as intermolecular "contacts." Despite the weakness of H-bond interactionsintermolecular bonds 100 to 1000 times weaker than those in ceramics or metals-these materials combine exceptional strength, robustness, and resilience. Individual $\mathrm{H}$ bonds behave like liquids, since their weak interactions can be disrupted even due to thermal fluctuations. Yet, materials such as spider silk and muscle fibers display great mechanical resistance against deformation and failure. This means for achieving high-strength materials from weak bonds is an intriguing question that has so far remained unanswered.

Atomistic simulation (Lu and Schulten, 2000; Lee et al., 2006) and single-molecule force spectroscopy studies (Rief et al., 1997; Oberhauser et al., 1998; Rief, Gautel, et al., 1998; Rief et al., 1999) have shown that beta-sheet rich proteins fail at particularly large forces, since they employ parallel strands with numerous $\mathrm{H}$ bonds that act as mechanical clamps under shear loading (Rohs et al., 1999; Brockwell et al., 2003; Grater et al., 2005; West et al., 2006). The unfolding behavior depends strongly on the rate of loading, as discussed (Ackbarow et al., 2007; Hyeon and Thirumalai, 2007; Sotomayor and Schulten, 2007). In earlier studies, the mechanical resistance of proteins has been linked to the orientation of betastrand domains with respect to the applied load (Rohs et al., 1999; Brockwell et al., 2003; Grater et al., 2005; West et al., 2006). However, how the number of $\mathrm{H}$ bonds in a strand (or equivalently the strand length) influences the strength has not been explained. For instance, universal structural features of protein chains are directly linked to the common native states and aggregation mechanisms of proteins (Banavar and Maritan, 2007; Knowles et al., 2007). For the inverse problem of unfolding, however, there exist only case specific phenomenological descriptions based on determining the energy barrier and the location of the transition state of mechanical unfolding pathways, extracted by studying the rate dependence of the mechanical unfolding force and the subsequent analysis with statistical theories. Linking this information to deformation mechanisms and the specific rupture events in the context of the unfolding of complex protein structures remains as a challenge. Similarly, although theoretical and experimental studies have provided great insight into the strength of multiple parallel hydrogen bonds (Evans and Ritchie, 1997; Heymann and Grubmuller, 2000; Seifert, 2000; Erdmann and Schwarz, 2004), the coupling between elasticity of the protein backbone and the rupture mechanisms of $\mathrm{H}$ bonds has not yet been clarified.

In silk and many other high-strength polymer materials the extreme mechanical stress conditions typically resemble a shear loading scenario in which the parallel arrangement of $\mathrm{H}$ bonds cooperatively resist force (Buehler et al., 2008; Keten et al., 2010); the cooperative action of groups of $\mathrm{H}$ bonds is the key to understand their ability to develop a significant mechanical strength. However, despite decades of research the molecular 
(a)

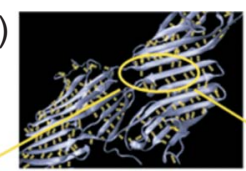

(b)

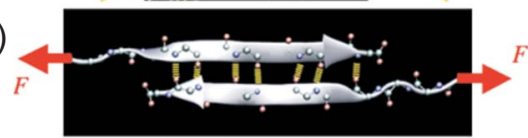

(c)

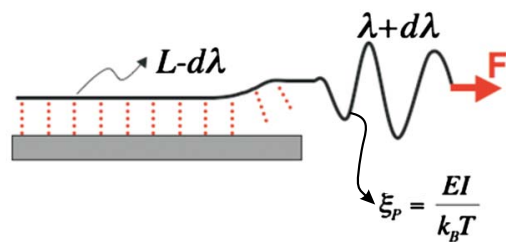

FIG. 17. (Color online) Example of a protein undergoing unfolding and the schematics for the theoretical model that represents a key resisting $\mathrm{H}$-bond cluster acting as a mechanical clamp (Keten and Buehler, 2008a). (a) A large beta-sheet protein consisting of H-bonded strands as shown in (b). (c) The schematic for the theoretical model presented in Sec. IV.C. Per unit rupture advancement $d \lambda$, an energy balance criterion between entropic elasticity and H-bond dissociation energy can be written to obtain the failure strength. Elasticity of the chain is modeled by the wormlike chain theory, and has one parameter, the persistence length $\xi_{P}$, that is linked to the bending rigidity of the polypeptide, EI. Note that the beta-strand also represents a fundamental constituent in spider silk, one of the toughest materials known (see Fig. 4).

mechanisms that make spider silk and similar materials so strong and yet extensible remains unknown. As a starting point, a fundamental question that applies to such protein materials that employ $\mathrm{H}$ bonds in a bottom-up design scheme is the level of maximum force that could be generated by a polypeptide chain stabilized by numerous $\mathrm{H}$ bonds.

In a simple approach, one can seek the maximum strength that can be achieved by a H-bond cluster as shown in Fig. 17 (Keten and Buehler, 2008a). Could the Griffith-Irwin (Griffith, 1921) criterion introduced earlier in context of continuum formulations be applied to peptide materials at the nanoscale for calculating the strength of a molecular assembly? The original idea stems from atomistic principles, namely, energetics of bond stretching and rupture; hence the model is indeed applicable with minor modifications. Since polypeptides predominantly exhibit nonlinear elasticity dominated by entropic effects, a free energy state equation (rather than potential energy) can describe the critical rupture condition. For polymers and polypeptides, the free energy balance criterion for failure can be stated as follows: The energy required to break $\mathrm{H}$ bonds equals the entropic elastic energy change due to stretching of the released polypeptide segment per unit length of rupture. The free energy state function of the system can be written as

$$
A(x, \lambda)=\lambda A_{\mathrm{WLC}}(x / \lambda)-F x-\gamma_{s}(L-\lambda),
$$

where $x$ and $\lambda$ are the end-to-end and contour length of the free chain, respectively, $L$ is the contour length of the bonded region, $\gamma_{s}$ is the adhesion strength of $\mathrm{H}$ bonds per unit length $\left(\gamma_{s}=E_{b} / L_{b}\right.$, ratio of H-bond dissociation energy $E_{b}$ to the H-bond spacing length $L_{b}$ ), $A_{\mathrm{WLC}}=\int_{0}^{\alpha} F_{\mathrm{WLC}}(\alpha) d \alpha$ denotes the free energy change due to stretching the chain based on the wormlike chain (WLC) model (Bustamante et al., 1994), and $F$ is the external applied force. The wormlike chain elasticity expression is given as

$$
F_{\mathrm{WLC}}=\frac{k_{B} T}{4 \xi_{P}}\left[(1-\alpha)^{-2}+4 \alpha-1\right]
$$

where $\alpha=x / \lambda$ denotes the stretch level of the chain and $\xi_{P}$ is the persistence length. Equation (17) represents a continuum approximation for the elasticity of polypeptide chains; any other appropriate elasticity model could also be used in context of the approach presented here.

We now assess the condition for rupture by taking derivative of Eq. (16) with respect to $\mathrm{d} \lambda ; d A / d \lambda=0$ yields the critical $\alpha$ value (denoted as $\alpha_{\text {cr }}$ ) that will enable propagation of bond rupture under constant force. The critical value $\alpha_{\text {cr }}$ can then be substituted into Eq. (17) to obtain the failure force $F_{\text {cr }}$. The force extension behavior showing energy dissipated in a unit cycle of bond rupture is shown in Fig. 18(a). Using typical established values for $\mathrm{H}$-bond strength and the persistence length of the protein's polypeptide backbone $\left(E_{b}\right.$ $=4 \mathrm{kcal} / \mathrm{mol}, \xi_{p}=0.4 \mathrm{~nm}$ ), we estimate the maximum strength of a uniformly loaded H-bonded assembly to be $F_{\mathrm{cr}}=127 \mathrm{pN}$. When the variation in bond energy or in the polypeptide chain elasticity is considered, for instance due to solvent conditions, this value ranges approximately from 100 to $300 \mathrm{pN}$ for relevant values of $E_{b}$ and $\xi_{P}$. This finding is in excellent agreement with a large number of AFM experiments at near-equilibrium rates on beta proteins, as shown in Fig. 18(b) (Sotomayor and Schulten, 2007; Keten and Buehler, 2008a, 2008c). This comparison shows that the simple fracture mechanics based model is applicable to describe a variety of protein rupture experiments.

The strength value developed previously, however, gives us no clue on how many $\mathrm{H}$ bonds are actually involved in the process of rupture; it only gives information on what force level is required to continuously break an assembly of $\mathrm{H}$ bonds in an irreversible fashion. How can we estimate the number of bonds that participate in the rupture process? A simple approach could be to use Bell's model which is an expression for describing how external force influences probability of bond rupture (Bell, 1978). According to Bell's formulation, one can formulate the influence of the external force on the effective energy barrier of a cluster of chemical bonds. For cooperative rupture of a group of $\mathrm{H}$ bonds, the effective energy barrier scales linearly with the number of bonds $N$, such that $E_{b, \text { eff }}=N E_{b}$, leading to the force prediction 
(a)

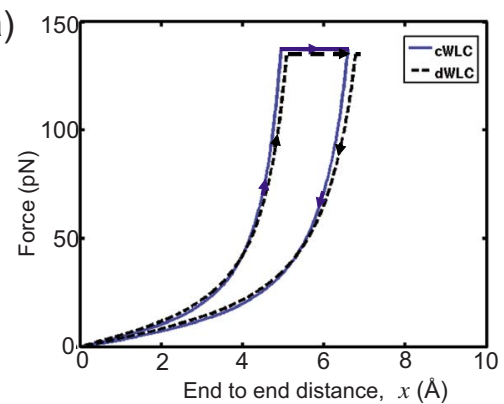

(b)

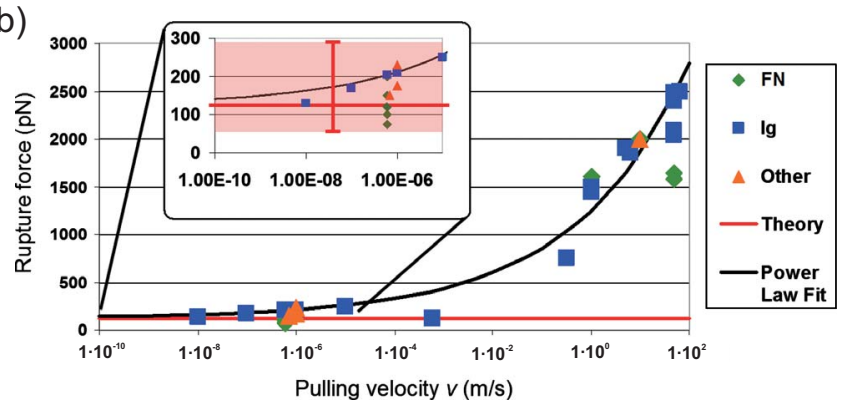

FIG. 18. (Color online) Strength model of H-bond clusters in beta-sheets, including a direct comparison between experiment and theory. (a) The force-extension behavior displayed before and after rupture, illustrating the dissipated energy (Keten and Buehler, 2008a). The continuum WLC (cWLC) expression used in the derivation could be replaced by discrete WLC (dWLC) formula proposed in Rosa et al. (2003) to see if there is any variation in the predicted strength. Both forms of the WLC expression predict similar elastic behavior and rupture force. (b) Summary of studies on rupture force of betadomains as a function of pulling rate. We summarize findings [adapted from Sulkowska and Cieplak (2007)] of the strength of beta-sheets rich proteins (focus on fibronectin, immunoglobin domains in ECM and titin). The overall behavior suggests that the rupture force asymptotically approaches a limiting value for vanishing pulling rates [continuous line is a power law fit to data for Ig27 (Sotomayor and Schulten, 2007)]. The inset shows a close-up of the strength limit and asymptotic behavior. The shaded region shows the range of values admissible by the theoretical prediction. The theoretical prediction range and experimental scattering of data show close agreement.

$$
F_{\mathrm{Bell}}(N)=\frac{1}{x_{B}}\left\{k_{B} T \ln \left[(\omega \tau)^{-1}\right]+N E_{b}\right\},
$$

where $x_{B}$ is the distance over which the force acts $\left(x_{b}\right.$ $\approx 4 \AA$, is derived from the geometry of $\mathrm{H}$ bonds loaded in shear in a beta strand; the force has to act over this distance to locally stretch and break the bonds simultaneously), $\omega$ is the natural frequency of bond vibration (on the order of $1 \times 10^{13} \mathrm{sec}^{-1}$ ), $\tau \approx 20 \mathrm{psec}$ is the characteristic time scale of bond rupture (Ackbarow et al., 2007), $N$ is the number of $\mathrm{H}$ bonds in the cluster considered, and $E_{b}$ is the dissociation energy of a single $\mathrm{H}$ bond. All parameters that appear in Eq. (18) can be estimated from fundamental geometric arguments, experiment, or molecular dynamics simulation. Using Eq. (18) inversely, one can link force acting over a certain time and distance to an approximate representative energy barrier that is overcome, which can then be linked to the number of hydrogen bonds broken in the process of rupture.

One can estimate the number of $\mathrm{H}$ bonds that are required to attain the critical rupture force, computed earlier as $F_{\text {cr }}$, by setting

$$
F_{\text {Bell }}\left(N_{\text {cr }}\right)=F_{\text {cr }}
$$

and solving for $N_{\text {cr }}$, the critical number of bonds that constitute the unit fracture mechanism in H-bonded polypeptides. For the values selected here, the theory predicts that the maximum number of bonds that participate in a single rupture event is limited to approximately four $\mathrm{H}$ bonds. This hypothesis has been further confirmed through molecular dynamics simulations (Keten and Buehler, 2008b). This prediction suggests that a cluster of four $\mathrm{H}$ bonds is optimal for achieving a high resistance to mechanical shear stress. Larger clusters would fail catastrophically at the same load level in a process similar to crack propagation, where a rupture process zone propagates through the beta strand, providing no additional mechanical stability. Figure 19 shows this concept by plotting the effective shear strength over the size of the H-bond cluster. This plot explains how it is possible to achieve a finite mechanical strength out of such a weak mechanical element by creating a particular geometric structure so that all $\mathrm{H}$ bonds in a cluster can cooperatively contribute to the strength. This analysis also confirms our initial conjecture that individual $\mathrm{H}$ bonds have no mechanical resistance (behave as liquids), and shows that by grouping them under geometric confinement a significant mechanical shear resistance can be achieved.

This finding becomes particularly intriguing when compared with typical size of H-bond clusters in beta sheets as found in biological materials. Recent proteomics data (Penel et al., 2003) suggest that long beta strands are highly disfavored in biology, and most common strand lengths are around four to six residues. Since each residue typically forms one $\mathrm{H}$ bond, these beta sheets typically employ four to six $\mathrm{H}$ bonds and are therefore in correlation with these results. This finding indicates that the driving forces that lead to folding and formation of thermodynamically stable beta sheets simultaneously account for maximum mechanical stability as well. Whether the relationship between mechanical and thermodynamical stability is a correlation or causality is of extreme importance for our fundamental understanding of the building blocks of life, and needs to be further investigated. When other protein structures, such as alpha helices or beta helices are considered, the typical H-bond clusters in a convolution or sheet also employ three to four $\mathrm{H}$ bonds, illustrating that geometric confinement of weak bonds within flexible bonded chains to short length scales may be a universal evolutionary design strategy that leads to the unique mechanical properties of protein materials. Figure 20 shows an overview over the typical size of H-bond clusters as found in natural protein structures. Another intriguing 
(a)

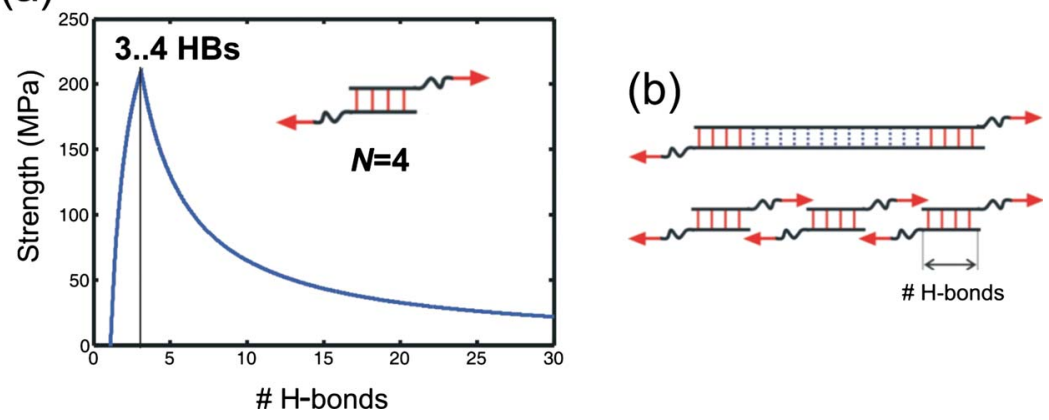

FIG. 19. (Color online) Turning weakness into strength: size dependence of hydrogen bond strength. Size effects of the shear strength of beta sheets [the geometry of a beta sheet is shown in the inlay of (a)] composed of two polypeptide chains connected through $N \mathrm{H}$ bonds; this number of $\mathrm{H}$ bonds has a significant effect on the shear strength of the structure (Keten and Buehler, 2008b). (a) The shear strength as a function of the number of $\mathrm{H}$ bonds of a beta-sheet structure (inset shows an example with four $\mathrm{H}$ bonds). (b) The physical significance of this size effect. In the upper plot, only $\mathrm{H}$ bonds at the boundary participate in the rupture process and provide resistance. In the lower plot, all $\mathrm{H}$ bonds throughout the entire structure contribute to the strength, making the overall structure three times stronger. The shear strength if defined as the strength of the beta strand divided by the sheared area.

aspect of this geometric confinement effect is that in materials such as spider silk, poly-alanine beta-sheet strand lengths are also six to seven residues, enabling the extreme strength and elasticity of these materials through nanoconfinement. Such materials typically employ small bond clusters that by themselves can resist hundreds of $\mathrm{pN}$ within $2-3 \mathrm{~nm}$ contact surfaces, possibly leading to

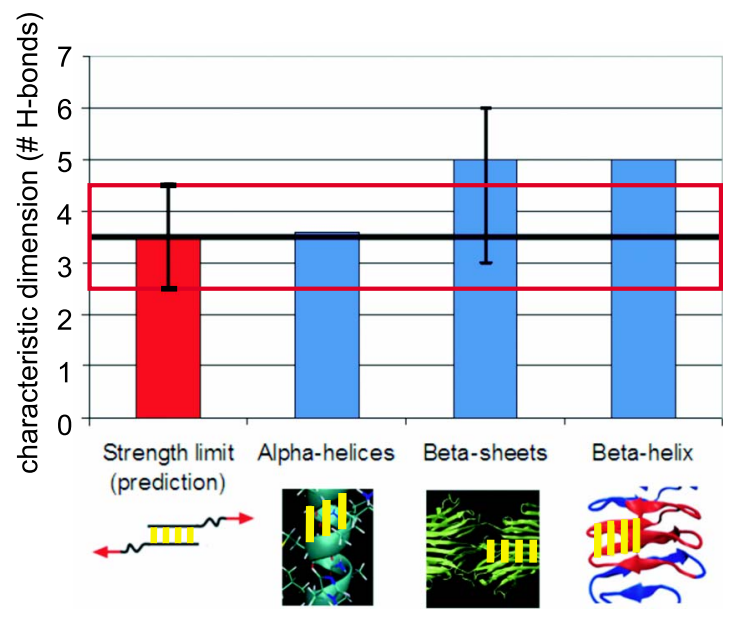

FIG. 20. (Color online) Characteristic dimensions of the size of $\mathrm{H}$-bond clusters in common protein structures (Keten and Buehler, 2008b). We compare the characteristic dimensions of alpha-helices, beta-sheets, and beta-helices to the dimensions associated with the optimal strength presented in Fig. 19 (see left bar, suggesting an optimal strength at three to four $\mathrm{H}$ bonds). Since the theoretical derivation reviewed here considers uniform deformation of hydrogen bonds with no particular specificity to geometry, it may also apply to other protein structures where nature utilizes geometric confinement to achieve higher mechanical stability. The fact that three to four $\mathrm{H}$ bonds per convolution exists on alpha-helices and beta-sheets on the sides of helices occur in clusters of approximately four may be indicative of such a universal biological concept that may be based on the evolutionary driving force to provide maximum strength. shear strengths that enable them to reach strengths comparable to metals (Vollrath and Knight, 2001). Future theoretical and computational studies on silklike nanostructural material constructs may lead to new design concepts for novel superfibers.

By considering the fracture mechanics of polypeptide H-bonded assemblies as a case study, here we have illustrated a general framework for describing molecular failure. The simplicity of the theory makes it quite generic and thus potentially applicable to a wide variety of nanostructured materials consisting of other protein secondary structures, nucleic acids, and polymers (Keten and Buehler, 2008a; Ackbarow, Keten, and Buehler, 2009). Further, such a simple theory is capable of explaining some of the interesting phenomena observed in experiments (Ainavarapu et al., 2008; Keten and Buehler, 2008a, 2008c).

\section{Failure of hierarchical materials: Putting it all together}

After discussing failure of structures at three distinct length scales, we now provide a discussion of failure properties of hierarchical materials. The hallmark feature of hierarchical materials is that they contain structures at multiple levels (see, e.g., Figs. 4 and 16). Due to the existence of multiple levels, failure of these materials can be particularly complex as each scale provides its own failure mechanism (and thus energy dissipation mechanism), which can be activated in a cascaded fashion. In fact, it has recently been discussed that the particular hierarchical structures found in biological materials could be the key to explain some of their most remarkable properties, such as the toughness of bone and nacre (seashells), or the stretchiness of spider silk and cells (Gao et al., 2003; Gao, 2006; Fratzl, 2007; Buehler et al., 2008; Espinosa et al., 2009).

The cascaded activation of mechanisms at multiple levels is a remarkable behavior ubiquitously found in biological materials that renders them capable to with- 
standing extreme deformation and large loads. We discuss in this section the key role multiscale mechanics plays in defining a material's ultimate response at failure, and how nature's design principles define the hierarchical structures of biological materials. This process, likely evolutionarily driven, enables materials to combine disparate properties such as strength and robustness (the ability to tolerate flaws), and adaptability to drastic changes in the environment.

The importance of hierarchical structures in defining a material's mechanical and fracture behavior was recently demonstrated (Ackbarow et al., 2009) for the case of alpha-helical protein networks as a model system for intermediate filaments (a key mechanical and structural component within a cell) whose hallmark is the extreme stretchiness of up to three to four times its initial length [Fig. 21(a)]. In this study, the cascaded activation of deformation mechanisms at multiple scales enables the material to tolerate structural flaws (cracks) of virtually any size. This unique behavior is in stark contrast to engineered materials (e.g., metals or ceramics; materials constructed with no hierarchies), where the presence of cracks leads to a severe reduction of strength and is the most common cause for catastrophic materials failure (Broberg, 1990). In conventional materials, failure typically initiates at locations of peak internal material stress at the corners of cracks, where atomic bonds are likely to break, leading to the propagation of fractures.

Unfortunately, flaws and cracks in materials cannot be avoided. The current engineering paradigm to address this issue is to overdimension materials, which has resulted in heavyweight structures where most of the excess material is never needed during regular operation. Biological materials, in contrast, show a different paradigm of materials design that involves an intrinsic ability to autonomously mitigate the adverse effects of material flaws (cracks) and are capable to render them innocuous, even to very large cracks. From a slightly different point of view, the generation of hierarchical structures can be seen as the deliberate placement of defects at controlled locations and scales (along with the creation of multiple material interfaces). Interestingly, it was demonstrated (Ackbarow et al., 2009) that the hierarchical makeup facilitated the dissipation of the local stress in the material by re-orientating a crack in an alpha-helical protein network under tension [Fig. 21(b)] from a horizontal to a vertical orientation, leading to a marginal increase in the stresses at the corners of the crack [Fig. 21(c)]. This change in the crack orientation provides a mechanism for the flawed material to deform several hundred percent and still avoid catastrophic failure, despite the presence of large flaws. Specifically, the plot includes a schematic of the crack corner stress concentration $\sigma_{\text {tip }}$ and the applied stress far away from the crack $\sigma_{0}$ [see also Fig. 3(b)]. The schematic shows that the initial horizontal crack orientation features a large stress concentration at the crack tip. In contrast, the transformed vertical crack orientation features only marginal stresses at its corners. (a)

H6
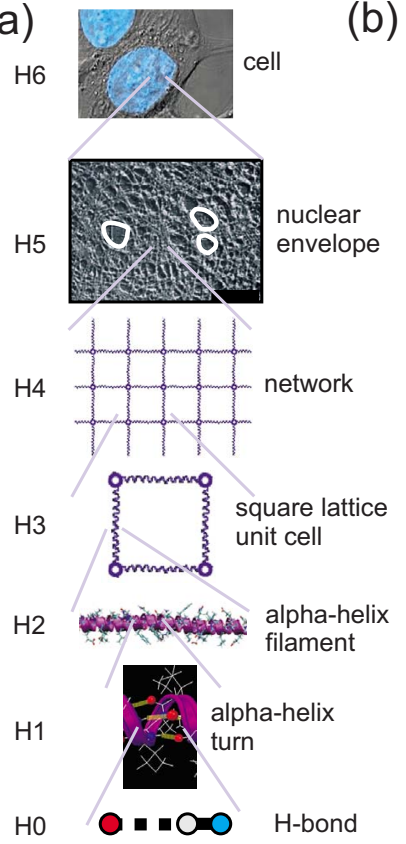

(b)

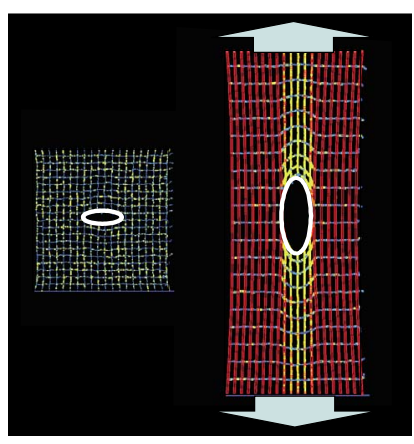

(c)

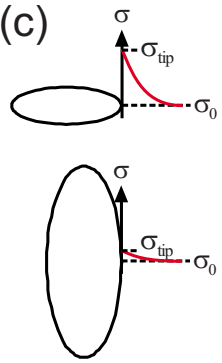

FIG. 21. (Color online) Hierarchical flaw tolerance mechanism in a cellular alpha-helix protein network (Ackbarow et al., 2009). (a) Seven levels of hierarchies are considered, from intrabackbone hydrogen bond ( $\mathrm{H} 0)$, alpha-helical turns $(\mathrm{H} 1)$, filaments of alpha-helices $(\mathrm{H} 2)$, to the representative unit cell (H3) of protein networks (H4) that form the cell nucleus (defects in the network highlighted) (H5) of eukaryotic cells (H6). The structure at each level is adapted to provide an optimal mechanical response and plays a key role in the overall mechanical behavior. Unfolding of alpha-helix turns (H1) proceeds via breaking of strong clusters of three to four $\mathrm{H}$ bonds (H0). The large deformation of alpha-helix filaments (with maximum strains of 100-200 \%) (H2) is enabled by the serial arrangements of many alpha-helical turns (H1). The severe stiffening of the filaments is enabled by alpha-to-beta-sheet transitions and backbone stretching, followed by interprotein sliding at the filament level $(\mathrm{H} 2)$, is a direct consequence of the structure of coiled alpha-helical proteins. The lattice structure (H3) is the key to facilitate large strain gradients in the protein network, enabling gigantic strain gradients at virtually no energetic cost at the network level (H4). This behavior is crucial for the flaw-tolerant behavior of the nuclear envelope level (H5), which is relevant to provide robust structural support to cells under large deformation (H6). (b) Protein network deformation with marked strain gradient, illustrating the change of the crack orientation from a horizontal to a vertical one. (c) Schematic of crack geometry transition, plotting the crack corner stress concentration $\sigma_{\text {tip }}$ and the applied stress far away from the crack $\sigma_{0}$. The schematic shows that the initial horizontal crack orientation features a large stress concentration at the crack tip. In contrast, the transformed vertical crack orientation features only marginal stresses at its corners.

The mechanisms that enable this intriguing behavior are intimately linked to the hierarchical structure. Seven levels of hierarchies are considered in the model system reviewed here, from intrabackbone hydrogen bond (H0), alpha-helical turns (H1), filaments of alpha helices $(\mathrm{H} 2)$, to the representative unit cell $(\mathrm{H} 3)$ of protein net- 
works (H4) that form the cell nucleus (defects in the network highlighted) (H5) of a eukaryotic cell (H6). Through using a bottom-up hierarchical multiscale simulation approach, it was shown that the structure at each level is adapted to provide an optimal mechanical response, and plays a key role in the overall mechanical behavior. Unfolding of alpha-helix turns (H1) proceeds via breaking of clusters of three to four $\mathrm{H}$ bonds $(\mathrm{H} 0)$, where the confinement to three to four $\mathrm{H}$ bonds is the key to maximize the mechanical strength (as discussed previously). The large deformation of alpha-helix filaments at constant force (with maximum strains of 100 $200 \%)(\mathrm{H} 2)$ is enabled by the serial arrangements of many alpha-helical turns (H1). The severe stiffening of the filaments is facilitated by alpha-to-beta-sheet transitions and backbone stretching, followed by interprotein sliding at the filament level (H2). These mechanisms, the alpha-to-beta-sheet transitions followed by backbone stretching, are a direct consequence of the structure of coiled alpha-helical proteins (Qin et al., 2009a, 2009b). At the next level, the lattice structure (H3) is the key to facilitate large strain gradients in the protein network, by enabling large strain gradients at virtually no energetic cost at the network level (H4). The formation of large strain gradients is essential to facilitate the rotation of the crack from the horizontal to the vertical orientation, as shown in Fig. 21(b). As shown in Fig. 21(c), this behavior is crucial for the flaw-tolerant behavior of the nuclear envelope level (H5), which is relevant to provide robust structural support to cells under large deformation (H6).

\section{MATERIALS FAILURE PHENOMENA IN THE CONTEXT OF DISEASE}

Throughout the past century, the focus on addressing diseases has derived primarily from a biochemical approach. However, advancements and increased understanding of quantitative measurements of materials phenomena at multiple scales has yielded an enhanced appreciation for the role of materials science of protein materials in variegated medical disorders. In this section, the role of biologically relevant material properties in the progression or activation of diseased states will be discussed.

Alzheimer's, Parkinson's, type II diabetes, and prion diseases (Selkoe, 2001; Hardy and Selkoe, 2002; Mesquida et al., 2007; Iconomidou and Hamodrakas, 2008) have been linked to the formation of foreign material deposits in tissues (also referred to as "ectopic materials" in the biomedical community). These material deposits, referred to as amyloid plaques, are highly ordered hierarchical assemblies of beta-sheet protein domains that form spontaneously (Burkoth et al., 2000; Dutt et al., 2005; Smith et al., 2006; Knowles et al., 2007). Once their formation is initiated, amyloid plaques grow uncontrollably to length scales of micrometers and persist under a wide range of $p \mathrm{H}$ conditions. The mechanical robustness of these plaques has been attributed to the large number of $\mathrm{H}$ bonds as well as to steric and hydrophobic interactions between different parts of the beta-sheet structures (Dobson, 2003; Chiti and Dobson, 2006; Knowles et al., 2007), albeit the exact structureprocess-property relationships for this material remain yet to be investigated. The properties of amyloid fibrils have been probed using AFM techniques and molecular dynamics simulations (Hwang et al., 2004; Mostaert et al., 2006; Smith et al., 2006; Ackbarow et al., 2007). It is believed that the formation of amyloid plaque deposits leads to neurotoxicity, which interferes with the biological function of the native tissue (Selkoe, 2001; Hardy and Selkoe, 2002). The mechanical robustness of amyloid plaques and the body's failure to eliminate these material deposits remains as a primary reason for our inability to reverse the progression of this disease. An analogy to the role of amyloids in neurodegenerative disease is the formation of sludge in combustion engines, where a lack of oil change leads to deposits of foreign materials in engines that may eventually lead to catastrophic failure. An improved understanding of how the hierarchical structure of amyloid plaques contribute to their extreme mechanical stability could lead to new strategies for treatment through targeting selective breakdown of these material deposits in situ.

Hutchinson-Gilford progeria syndrome, a genetic rapid aging disease, is caused by a structural defect in the lamin nuclear membrane due to changes in the amino acid sequence (Burke and Stewart, 2002; Lammerding et al., 2004; Gruenbaum et al., 2005; Dahl et al., 2006). The progression of this disease has been associated with mechanical failure of the cell's nuclear membrane in tissues subject to mechanical loading. A recent study based on live-cell imaging and micropipette aspiration has shown that progeria nuclear membranes display a reduced deformability and feature the formation of fractures upon application of mechanical load (Dahl, Scaffidi, et al., 2006). The cause of these fractures is attributed to changes in the lamin microstructure, where filaments form more ordered domains that prevent the dissipation of mechanical stress (where the number of filaments is not altered significantly, but their structural molecular makeup leads to changes in the microstructure). This structural alteration leads to a change in the deformation mechanism, from a dissipative mode ("ductile") in healthy cells to a catastrophic localized failure mode ("brittle") in diseased cells (see Fig. 22). These mechanisms appear predominantly in cells that are subjected to mechanical deformation, particularly in endothelial and smooth muscle cells of the vascular system. Perhaps a loss of mechanical integrity in the cell's nuclear membrane can influence gene regulation by triggering a wide range of biochemical processes that lead to the rapid aging phenomenon. However, the exact molecular failure mechanisms remain unknown and its investigation represents an opportunity for future research where a materials science approach could make important contribution. This disease illustrates how material failure due to structural flaws within a protein material can lead to the breakdown of critical biological components. Many other genetic diseases resulting from struc- 
(a)

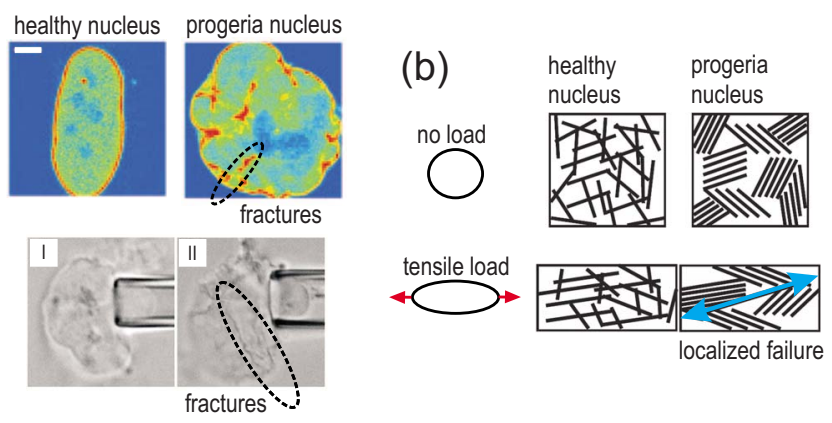

FIG. 22. (Color online) Role of changes in biological protein material properties in diseases, here exemplified for the rapid aging disease progeria. This shows how changes in the lamin microstructure due to the genetic mutation and effect on deformation mechanism. (a) A structural analysis of a healthy nucleus compared with a disease nucleus; showing that the progeria nucleus is more brittle and shows the formation of several small fractures. The lower part shows micropipetting experiments, also suggesting a more brittle behavior of the progeria nucleus. (b) A possible schematic of the mechanism behind these observations, suggesting a structural change of the protein filament microstructure due to the mutation. It is noted that the number of filaments is not altered significantly, but their structural molecular makeup leads to changes in the microstructure. Adapted from Dahl et al., 2006.

tural flaws in the lamin protein network have been identified, generally referred to as "laminopathies" (Burke and Stewart, 2002; Lammerding et al., 2004; Gruenbaum et al., 2005).

Other genetic disorders in collagenous tissues have been linked to the alteration of the material structure due to mutations in the genes that encode the tropocollagen molecule. Osteogenesis imperfecta is a genetic disease that enhances bone's susceptibility to catastrophic brittle fracture, a disease also referred to as "brittle bone disease." The origin of this disease resides in changes to the structure of tropocollagen molecules due to the substitution of a single glycine amino acid [Fig. 23(a)] (Byers et al., 1991; Prockop and Kivirikko, 1995). Some collagen mutations prevent the formation of triple helical molecules (procollagen suicide), while other mutations cause structural changes to tropocollagen molecules, leading to bending (e.g., due to kinks induced by amino acid substitutions), reduced mechanical stiffness (e.g., due to changes of the volume and hydrophibicity), or changes in the intermolecular adhesion (e.g., due to changes in surface charges) (Byers et al., 1991; Prockop and Kivirikko, 1995; Gautieri, Uzel, et al., 2009; Gautieri, Vesentini, et al., 2009). At mesoscopic length scales, these molecular-level changes lead to poor fibril packing (McBride et al., 1997; Miller et al., 2007) and a decrease in cross-link density (Sims et al., 2003; Miller et al., 2007). Changes in the size and shape of mineral crystals in bone (e.g., less organized, more round-shaped crystals) have also been reported (Fratzl et al., 1996; Camacho et al., 1999; Grabner et al., 2001), which might be related to a change in the ability of tropocollagen to bind to the (a)

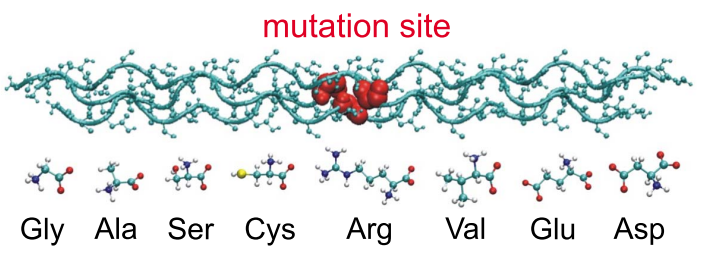

(b)
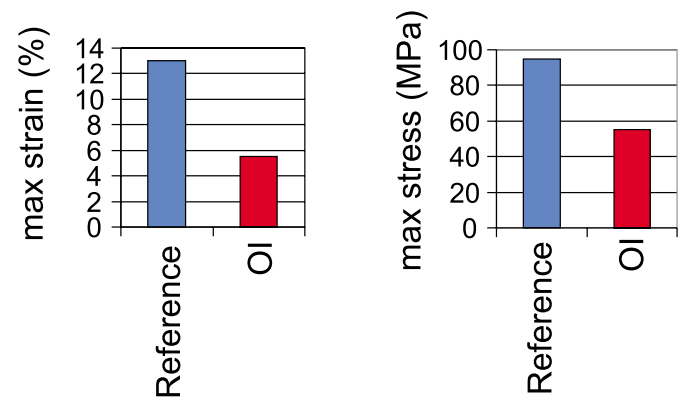

(c)
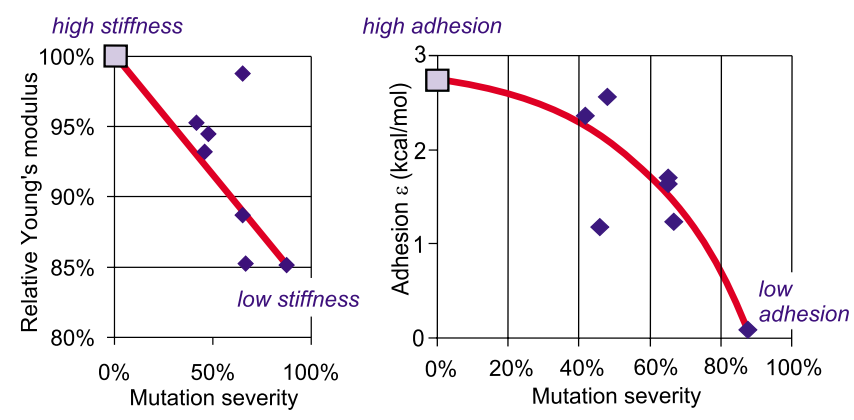

reference mutated

FIG. 23. (Color online) Mutations in osteogenesis imperfecta disease and impact on mechanics properties. Geometry of point mutations in osteogenesis imperfecta (brittle bone disease, often abbreviated as OI) in (a) the tropocollagen molecule and (b) effect on mechanical properties of tendon. The analysis shows that the maximum strain and maximum stress of tendon is severely reduced under mutations (lower part, data plotted from Misof, Landis, et al., 1997). The graphs shown in (c) illustrate that the relative strength of the effect of different mutations on the molecular stiffness and similarly, the intermolecular adhesion, correlates directly with the clinically measured severity of various types of mutations (Gautieri et al., 2009).

mineral phase of bone (Rauch and Glorieux, 2004; Miller et al., 2007). At larger length scales, the effects of osteogenesis imperfecta mutations lead to inferior mechanical properties of tendon and bone (Misof et al., 1997) [Fig. 23(b)]. A mechanically inferior collagen matrix in addition to an increased and less organized mineral content, and an overall reduced bone volume due to reduced bone turnover (Chavassieux et al., 2007), might explain the phenomenon of brittle bones, an important feature of osteogenisis imperfecta (Miller et al., 2007). Figure 24 shows the results of the influence of osteogenesis imperfecta mutations on the mechanical properties of a collagen fibril as obtained from molecular dynamics simulations, leading to a significant reduction of mechanical strength and yield strain (for most severe mutation, located at the end of the molecule) (Gautieri, Uzel, 

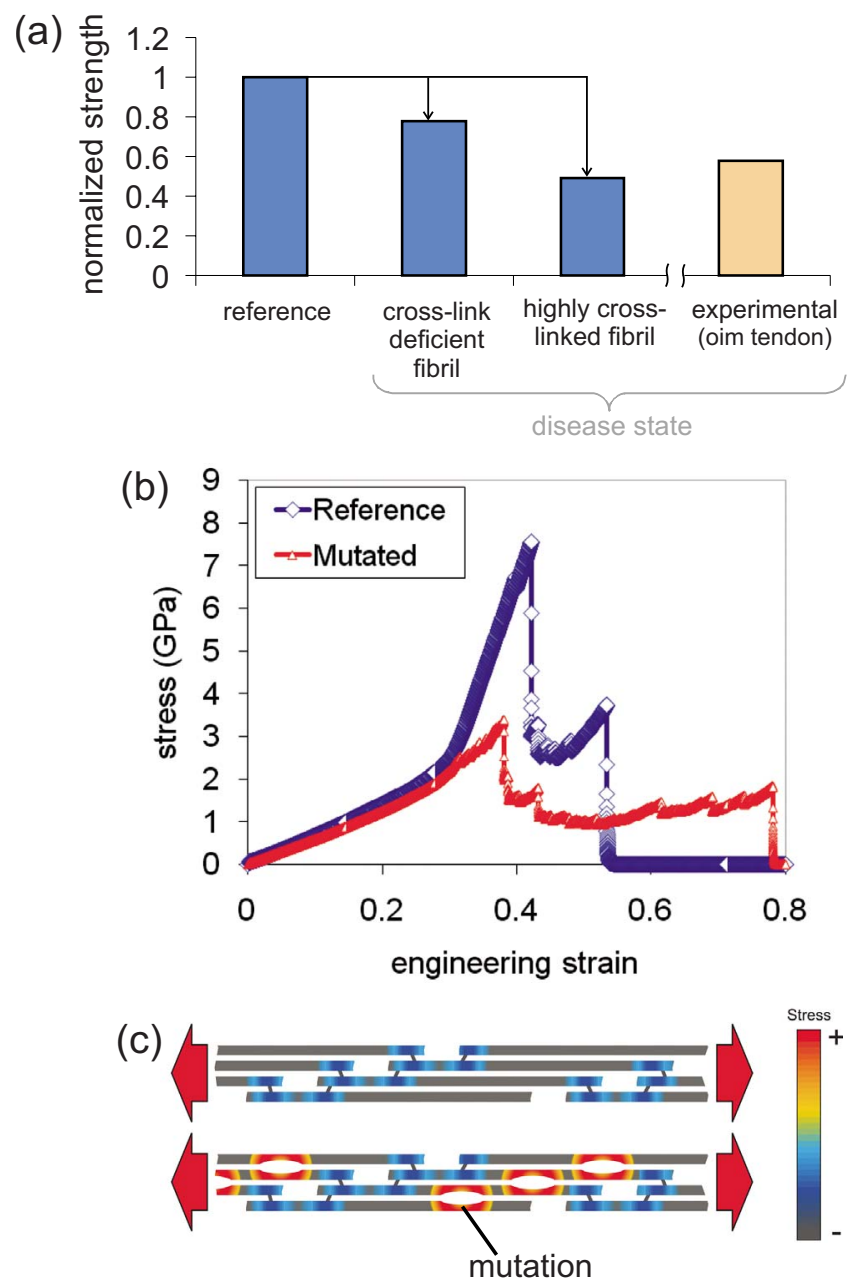

FIG. 24. (Color online) Influence of osteogenesis imperfecta mutations on the mechanical properties of a collagen fibril, leading to a significant reduction of mechanical strength and yield strain (for most severe mutation, located at the end of the molecule) (Gautieri, Uzel, et al., 2009). (a) The decrease of the strength for a mutation (in a cross-link deficient fibril and highly cross-linked fibril), including a qualitative comparison with experimental results (Misof et al., 1997) in osteogenesis imperfecta tendon (note the difference in scale; the MD studies are focused on single collagen fibrils; the experiments have been carried out at much larger tendon tissue scales). (b) The stress-strain curve for the reference case and a mutated case, showing that the presence of mutations can severely influence the overall mechanical signature (highly cross-linked fibril). Intermolecular gliding sets in at $30 \%$ vs $42 \%$ in the mutated fibril, and the maximum stress is significantly reduced (to about $50 \%$ of its reference value). The small-deformation elastic modulus is reduced by approximately $15 \%$ under the presence of the osteogenesis imperfecta mutation, due the formation of internal stress concentrations between collagen molecules at the sites of the mutations. (c) A schematic of the stress concentrations caused at the mutation sites. The formation of stress concentrations within collagen fibrils suggests that the breakdown of the homogeneous stress state as achieved in the healthy state (Buehler, 2006) results in a severe reduction of the strength and toughness of the material.

et al., 2009; Gautieri, Vesentini, et al., 2009). Figure 24(a) shows the decrease of the strength for a mutation (in a cross-link deficient fibril and highly cross-linked fibril), including a qualitative comparison with experimental results (Misof et al., 1997) in osteogenesis imperfecta tendon (note the difference in scale; the molecular simulation studies are focused on single collagen fibrils; the experiments have been carried out at much larger tendon tissue scales). Figure 24(b) shows the stress-strain curve for the reference case and a mutated case, showing that the presence of mutations can severely influence the overall mechanical signature (highly cross-linked fibril). Intermolecular gliding sets in at $30 \%$ vs $42 \%$ in the mutated fibril, and the maximum stress is significantly reduced (to about $50 \%$ of its reference value). The small-deformation elastic modulus is reduced by approximately $15 \%$ under the presence of the osteogenesis imperfecta mutation. The reason for the reduced strength of mutated fibrils is that the presence of the mutation induces a change of the stress field within the fibril; causing a magnification of the stress at the points of mutations. This is because mutations severely reduce the intermolecular adhesion, thus creating small interfacial cracklike defects inside the tissue that lead to local stress concentrations. The phenomenon is quite similar to the one known from cracks in solids as shown in Fig. 3(b), where local stress concentrations at defects can explain the sudden growth of cracks. Further research is needed to better understand these disease mechanisms and how they relate to materials failure phenomena under varying conditions. However, the examples discussed here illustrate that a bottom-up simulation approach, used together with simulation and theory, can be a powerful tool in investigating the initiation and progression of diseases and related materials mechanisms.

The examples also illustrate that the change of material properties and associated failure of a biological system is a crucial element in many diseases. The translation of this knowledge would enable detection of diseases by measuring material properties rather than by focusing on symptomatic biochemical readings alone. Close coupling of biochemical make-up, structural arrangement, and mechanical properties at the nanoscale make molecular modeling tools and indispensable tool for understanding disease and failure. Altogether, understanding the role of different hierarchical levels of protein materials in diseases could potentially bring about a new paradigm of approaches to address medical disorders; however, further research is needed to elucidate the underlying multiscale failure mechanisms. Biology utilizes hierarchical structures in an intriguing way to create multifunctional materials and illustrates how weakness can be turned into strength as in the case of the utilization of $\mathrm{H}$ bonds (Keten et al., 2010). Even though biochemical and image-based diagnostics will remain important the integration of scales, as well as the mixing of physical, biological, and chemical concepts into novel engineering designs could complement the current practice of disease diagnosis and treatment, as well as the design of new materials, and thereby unfold many opportunities for technological innovations. 


\section{DISCUSSION}

Materials failure processes are found in association with many types of materials and at multiple scales. Understanding failure is the key to success, since they are crucial to enable engineering and technological advances and to maintain and foster human civilization. As shown through various examples in this paper, failure of materials is a true multiscale phenomenon, where small microscopic mechanisms (often involving only a few atoms) translate into catastrophic failure at much larger scales. Several examples have been provided in this Colloquium, ranging from failure of a single crystal of silicon due to defects such as cracks (Fig. 6), failure of the Earth's crust in earthquakes as tectonic plates shear against each other (Fig. 10), failure of bone in injury (Fig. 15), to failure of molecules where the fundamental biological cement-H bonds-break and cause major structural changes in proteins (Fig. 17). It was also discussed that hierarchical materials can feature intriguing failure properties, such as the ability to tolerate flaws via built-in mechanisms to autonomously mitigate the adverse effects of cracks (Fig. 21). The concept of designing materials with hierarchical structures, by deliberately determining a cascade of multiscale mechanisms is a largely unexplored aspect in materials science that could lead to advances in de novo materials design. By utilizing self-assembly processes from nano to macro (Reches and Gazit, 2007), hierarchical structures may be the key that will enable us to take advantage of properties at all scales, and to exploit superior nanoscale properties through disparate scales (Buehler, 2010). Failure of cells (Fig. 22) and tissues (Figs. 23 and 24) in genetic disease has also been linked to the existence of defects. In all examples, the catastrophic failure of the system can be linked to microscopic (or multiscale) mechanisms that govern the overall material behavior.

Another core message that can be taken away from this Colloquium is that simplified theoretical approaches that capture the essential elements of a system are powerful as they build upon fundamental concepts. As a consequence, they are versatile and generally have implications for a wide range of systems that may even belong to vastly different length scales. The same is true for molecular simulations, as they rely on fundamentals of quantum chemistry (how chemical bonds behave) and depend less on case-specific empirical assumptions as required in phenomenological theories of materials. This has rendered the use of molecular simulation a powerful approach capable of predicting and revealing universal molecular mechanisms that govern a variety of physical phenomena that have been observed experimentally.

A particularly interesting area of application is the study of failure of biological systems, as discussed in Sec. $\mathrm{V}$, and illustrated for two genetic diseases (see Figs. 22-24) in which small genetic mutations (defects in the amino acid makeup) make a large difference in the overall system behavior. The wide impact of the use of materials science approaches in biology and biomedical sciences has yet to be demonstrated. In some specific areas such as bone (e.g., in the context of osteoporosis), cartilage (e.g., arthritis), cardiovascular aspects and, particularly, in the context of tissue engineering and regenerative medicine, materials science approaches have started to play an important role. In addition to mechanical deformation and failure mechanisms of protein materials in extreme conditions and disease, the investigation of other material properties could be of great interest as well. Optical properties (e.g., in the eye's cornea and lens), photoelectric properties (e.g., photosynthesis in plants), electrical (e.g., links between neuron cellssynapses), motility (e.g., in muscle tissues), or thermal properties (e.g., thermal management of biological organisms) are critically important issues that could be studied using a materials science approach, to systematically link processes, structure and properties. As for mechanical properties, the study of materials failure could provide an interesting platform to advance our understanding of diseases.

The study of failure of materials and structures is important for many other aspects. For example, whereas failure in biology is linked to disease, in nanotechnology the interaction of stable elements with biological cells and tissues is important for function but also in light of critical issues such as toxicity. In biotechnology, issues such as elasticity or deformation are linked to key biological processes. Mechanical compliance of nanoscale and macroscale implants and devices with surrounding soft tissue and bone is crucial as degradation rate and stiffness of implants highly influence biochemical signaling processes at cell and tissue level, governing the likelihood of major side effects and in some cases failure of organs. In aerospace and defense applications, there is a need for materials that enable longer missions in extreme environments (e.g., as part of space exploration). In such very expensive missions, the failure of a simple component jeopardizes the entire project, requiring stable and robust materials. Recent examples of infrastructure failure, such as the 2008 Minnesota bridge collapse, show that it is crucial to identify better materials (e.g., new types of concrete and steel) and also new methods to predict and detect accurate degradation and failure conditions ahead of time. Most commonly used building materials are associated with a large environmental footprint, as they require enormous amounts of energy during their production. For example, cement as the material most produced on our planet accounts for almost $10 \%$ of the human caused $\mathrm{CO}_{2}$ release (Pellenq et al., 2009). Designing new infrastructure materials that can be produced with less energy, at lower temperature, faster and with greater robustness and perhaps adaptability to account for changes in operational conditions is a new frontier in enabling and sustaining human civilization. For example, could the tools of genetic engineering of plants, currently applied to improve the quality and quantity of crops, be applied to engineer new types of wood or plant fibers with superior mechanical properties?

For all of these applications, cross-scale multiscale effects will be important as we push the limits of what we 
can see and how small and how effective we can design. For efficiency and conservation of finite resources, novel multiscale modeling methods will be required that enable us to explore the full design space, from nano to macro. New interatomic potentials that can accurately describe the breaking of bonds ( $\mathrm{H}$ bonds, covalent bonds, etc.) in a seamless multiscale scheme are needed to include the full complexity of chemical bonding in a numerically efficient description. New types of simplified models and approaches that bridge the knowledge between different engineering and scientific disciplines are necessary, and may lead to emerging fields with huge potential impact for society and technological advancement.

\section{ACKNOWLEDGMENTS}

This research was supported by the Army Research Office (W911NF-06-1-0291), by the National Science Foundation (CAREER Grant CMMI-0642545 and MRSEC DMR-0819762), by the Air Force Office of Scientific Research (FA9550-08-1-0321), by the Office of Naval Research (N00014-08-1-00844), and by the Defense Advanced Research Projects Agency (DARPA) (HR0011-08-1-0067). M.J.B. acknowledges support through the Esther and Harold E. Edgerton Career Development Professorship.

\section{REFERENCES}

Ackbarow, T., X. Chen, S. Keten, and M. J. Buehler, 2007, "Hierarchies, multiple energy barriers, and robustness govern the fracture mechanics of alpha-helical and beta-sheet protein domains," Proc. Natl. Acad. Sci. U.S.A. 104, 16410.

Ackbarow, T., S. Keten, and M. J. Buehler, 2009, "A multitimescale strength model of alpha-helical protein domains," J. Phys.: Condens. Matter 21, 035111-7.

Ackbarow, T., D. Sen, C. Thaulow, and M. J. Buehler, 2009, "Alpha-helical protein networks are self protective and flaw tolerant," PLoS ONE 4, e6015.

Ainavarapu, S. R. K., A. P. Wiita, L. Dougan, E. Uggerud, and J. M. Fernandez, 2008, "Single-molecule force spectroscopy measurements of bond elongation during a bimolecular reaction," J. Am. Chem. Soc. 130, 6479.

Aizenberg, J., J. C. Weaver, M. S. Thanawala, V. C. Sundar, D. E. Morse, and P. Fratzl, 2005, "Skeleton of Euplectella sp.: Structural hierarchy from the nanoscale to the macroscale," Science 309, 275.

Alava, M. J., P. K. V. V. Nukalaz, and S. Zapperi, 2006, "Statistical models of fracture," Adv. Phys. 55, 349.

Alberts, B., A. Johnson, J. Lewis, M. Raff, K. Roberts, and P. Walter, 2002, Molecular Biology of the Cell (Taylor \& Francis, New York).

Andrews, D. J., 1976, "Rupture velocity of plane strain shear cracks,” J. Geophys. Res. 81, 5679.

Arkhipov, A., P. L. Freddolino, K. Imada, K. Namba, and K. Schulten, 2006, "Coarse-grained molecular dynamics simulations of a rotating bacterial flagellum," Biophys. J. 91, 4589.

Bahar, I., and R. Jernigan, 1997, "Inter-residue potentials in globular proteins and the dominance of highly specific hydrophilic interactions at close separation,” J. Mol. Biol. 266, 195.
Bak, P., C. Tang, and K. Wiesenfeld, 1988, "Self-organized criticality," Phys. Rev. A 38, 364.

Banavar, J. R., and A. Maritan, 2007, "Physics of proteins," Annu. Rev. Biophys. Biomol. Struct. 36, 261.

Bathe, M., 2008, "A finite element framework for computation of protein normal modes and mechanical response," Proteins: Struct., Funct., Bioinf. 70, 1595.

Bazant, Z. P., 1999, "Size effect on structural strength: A review," Arch. Appl. Mech. 69, 703.

Bell, G. I., 1978, "Models for specific adhesion of cells to cells," Science 200, 618.

Brenner, D. W., O. A. Shenderova, J. A. Harrison, S. J. Stuart, B. Ni, and S. B. Sinnott, 2002, "A second-generation reactive empirical bond order (REBO) potential energy expression for hydrocarbons," J. Phys.: Condens. Matter 14, 783.

Broberg, K. B., 1990, Cracks and Fracture (Academic, New York).

Brockwell, D. J., E. Paci, R. C. Zinober, G. S. Beddard, P. D. Olmsted, D. A. Smith, R. N. Perham, and S. E. Radford, 2003, "Pulling geometry defines the mechanical resistance of a beta-sheet protein," Nat. Struct. Biol. 10, 731.

Buehler, M. J., 2006, "Nature designs tough collagen: Explaining the nanostructure of collagen fibrils," Proc. Natl. Acad. Sci. U.S.A. 103, 12285.

Buehler, M. J., 2007a, "Molecular nanomechanics of nascent bone: Fibrillar toughening by mineralization," Nanotechnology 18, 295102-9.

Buehler, M. J., 2007b, "Hierarchical chemo-nanomechanics of stretching protein molecules: Entropic elasticity, protein unfolding and molecular fracture," J. Mech. Mater. Struct. 2, 1019.

Buehler, M. J., 2008, Atomistic Modeling of Materials Failure (Springer, New York).

Buehler, M. J., 2010, "Strength in numbers," Nat. Nanotechnol. 5, 172.

Buehler, M. J., F. F. Abraham, and H. Gao, 2003, "Hyperelasticity governs dynamic fracture at a critical length scale," Nature (London) 426, 141.

Buehler, M. J., J. Dodson, P. Meulbroek, A. Duin, and W. A. Goddard, 2006, "The Computational Materials Design Facility (CMDF): A powerful framework for multiparadigm multiscale simulations," Mater. Res. Soc. Symp. Proc. 894, LL3.8. Buehler, M. J., A. C. T. v. Duin, and W. A. Goddard, 2006, "Multiparadigm modeling of dynamical crack propagation in silicon using a reactive force field," Phys. Rev. Lett. 96, 095505.

Buehler, M. J., and H. Gao, 2006a, Ultra Large Scale Atomistic Simulations of Dynamic Fracture Handbook of Theoretical and Computational Nanotechnology, edited by W. Schommers and A. Rieth (American Scientific Publishers, Stevenson Ranch, CA).

Buehler, M. J., and H. J. Gao, 2006b, "Dynamical fracture instabilities due to local hyperelasticity at crack tips," Nature (London) 439, 307.

Buehler, M. J., S. Keten, and T. Ackbarow, 2008, "Theoretical and computational hierarchical nanomechanics of protein materials: Deformation and fracture," Prog. Mater. Sci. 53, 1101.

Buehler, M. J., H. Tang, A. C. T. v. Duin, and W. A. Goddard, 2007, "Threshold crack speed controls dynamical fracture of silicon single crystals," Phys. Rev. Lett. 99, 165502.

Buehler, M. J., and Y. C. Yung, 2009, "Deformation and failure of protein materials in physiologically extreme conditions and 
disease," Nature Mater. 8, 175.

Buehler, M. J., and Z. Xu, 2010, "Mind the helical crack," Nature (London) 464, 42.

Burke, B., and C. L. Stewart, 2002, "Life at the edge: The nuclear envelope and human disease," Nat. Rev. Mol. Cell Biol. 3, 575.

Burkoth, T. S., T. L. S. Benzinger, V. Urban, D. M. Morgan, D. M. Gregory, P. Thiyagarajan, R. E. Botto, S. C. Meredith, and D. G. Lynn, 2000, "Structure of the beta-amyloid((10-35)) fibril," J. Am. Chem. Soc. 122, 7883.

Burridge, R., 1973, "Admissible speeds for plain-strain selfsimilar cracks with friction but lacking cohesion," Geophys. J. R. Astron. Soc. 35, 439.

Bustamante, C., J. F. Marko, E. D. Siggia, and S. Smith, 1994, "Entropic elasticity of lambda-phage DNA," Science 265, 1599.

Byers, P. H., G. A. Wallis, and M. C. Willing, 1991, "Osteogenesis imperfecta: Translation of mutation to phenotype," J. Med. Genet. 28, 433.

Camacho, N. P., L. Hou, T. R. Toledano, W. A. Ilg, C. F. Brayton, C. L. Raggio, L. Root, and A. L. Boskey, 1999, "The material basis for reduced mechanical properties in oim bones," J. Bone Miner. Res. 14, 264.

Chavassieux, P., E. Seeman, and P. D. Delmas, 2007, "Insights into material and structural basis of bone fragility from diseases associated with fractures: How determinants of the biomechanical properties of bone are compromised by disease," Endocr. Rev. 28, 151.

Chenoweth, K., S. Cheung, A. C. T. van Duin, W. A. Goddard, and E. M. Kober, 2005, "Simulations on the thermal decomposition of a poly(dimethylsiloxane) polymer using the $\mathrm{Re}$ axFF reactive force field," J. Am. Chem. Soc. 127, 7192.

Cheung, S., W. Q. Deng, A. C. T. van Duin, and W. A. Goddard, 2005, "ReaxFF( $\mathrm{MgH})$ reactive force field for magnesium hydride systems," J. Phys. Chem. A 109, 851.

Chiti, F., and C. M. Dobson, 2006, "Protein misfolding, functional amyloid, and human disease," Annu. Rev. Biochem. 75, 333.

Dahl, K. N., P. Scaffidi, M. F. Islam, A. G. Yodh, K. L. Wilson, and T. Misteli, 2006, "Distinct structural and mechanical properties of the nuclear lamina in Hutchinson-Gilford progeria syndrome," Proc. Natl. Acad. Sci. U.S.A. 103, 10271.

Deniz, A. A., S. Mukhopadhyay, and E. A. Lemke, 2008, "Single-molecule biophysics: at the interface of biology, physics and chemistry," J. R. Soc., Interface 5, 15.

Dietz, H., and M. Rief, 2008, "Elastic bond network model for protein unfolding mechanics,” Phys. Rev. Lett. 100, 098101.

Diez-Perez, A., R. Güerri, X. Nogues, E. Cáceres, M. J. Peña, L. Mellibovsky, C. Randall, D. Bridges, J. C. Weaver, A. Proctor, D. Brimer, K. J. Koester, R. O. Ritchie, and P. K. Hansma, 2010, "Microindentation for in vivo measurement of bone tissue mechanical properties in humans," J Bone Min. Res. (to be published).

Dobson, C. M., 2003, "Protein folding and misfolding," Nature (London) 426, 884.

Duin, A. C. T. v., S. Dasgupta, F. Lorant, and W. A. Goddard, 2001, "ReaxFF: A reactive rorce field for hydrocarbons," J. Phys. Chem. A 105, 9396.

Duin, A. C. T. v., A. Strachan, S. Stewman, Q. Zhang, X. Xu, and W. A. Goddard, 2003, "ReaxFF SiO: Reactive force field for silicon and silicon oxide systems," J. Phys. Chem. A 107, 3803.

Dutt, A., M. G. B. Drew, and A. Pramanik, 2005, "Beta-sheet mediated self-assembly of dipeptides of Omega-amino acids and remarkable fibrillation in the solid state," Org. Biomol. Chem. 3, 2250.

Erdmann, T., and U. S. Schwarz, 2004, "Stability of adhesion clusters under constant force," Phys. Rev. Lett. 92, 108102.

Espinosa, H. D., J. E. Rim, F. Barthelat, and M. J. Buehler, 2009, "Merger of structure and material in nacre and bonePerspectives on de novo biomimetic materials," Prog. Mater. Sci. 54, 1059.

Evans, E., and K. Ritchie, 1997, "Dynamic strength of molecular adhesion bonds,” Biophys. J. 72, 1541.

Fratzl, P., 2007, "Biomimetic materials research: what can we really learn from nature's structural materials?" J. R. Soc., Interface 4, 637.

Fratzl, P., O. Paris, K. Klaushofer, and W. J. Landis, 1996, "Bone mineralization in an osteogenesis imperfecta mouse model studied by small-angle X-ray scattering," J. Clin. Invest. 97, 396.

Fratzl, P., and R. Weinkamer, 2007, "Nature's hierarchical materials,” Prog. Mater. Sci. 52, 1263.

Freund, L. B., 1990, Dynamic Fracture Mechanics (Cambridge University Press, Cambridge).

Gao, H., 1996, "A theory of local limiting speed in dynamic fracture," J. Mech. Phys. Solids 44, 1453.

Gao, H., Y. Huang, and F. F. Abraham, 2001, "Continuum and atomistic studies of intersonic crack propagation," J. Mech. Phys. Solids 49, 2113.

Gao, H., B. Ji, I. Jager, E. Arzt, and P. Fratzl, 2003, "Materials become insensitive to flaws at nanoscale: Lessons from nature," Proc. Natl. Acad. Sci. U.S.A. 100, 5597.

Gao, H. J., 2006, “Application of fracture mechanics concepts to hierarchical biomechanics of bone and bone-like materials," Int. J. Fract. 138, 101.

Garcimartin, A., A. Guarino, L. Bellon, and S. Ciliberto, 1997, "Statistical properties of fracture precursors," Phys. Rev. Lett. 79, 3202.

Gautieri, A., S. Uzel, S. Vesentini, A. Redaelli, and M. J. Buehler, 2009, "Molecular and mesoscale mechanisms of osteogenesis imperfecta disease in collagen fibrils," Biophys. J. 97, 857.

Gautieri, A., S. Vesentini, A. Redaelli, and M. J. Buehler, 2009, "Single molecule effects of osteogenesis imperfecta mutations in tropocollagen protein domains," Protein Sci. 18, 161.

Gerde, E., and M. Marder, 2001, "Friction and fracture," Nature (London) 413, 285.

Goddard, W. A., 2006, in A Perspective of Materials Modeling Handbook of Materials Modeling, edited by S. Yip (Springer, New York).

Grabner, B., W. J. Landis, P. Roschger, S. Rinnerthaler, H. Peterlik, K. Klaushofer, and P. Fratzl, 2001, "Age- and genotype-dependence of bone material properies in the osteogenesis imperfecta murine model (oim)," Bone (N.Y.) 29, 453.

Grater, F., J. Shen, H. Jiang, M. Gautel, and H. Grubmuller, 2005, "Mechanically induced titin kinase activation studied by force-probe molecular dynamics simulations," Biophys. J. 88, 790.

Griffith, A. A., 1921, "The phenomena of rupture and flow in solids," Philos. Trans. R. Soc. London, Ser. A 221, 163.

Gruenbaum, Y., A. Margalit, R. D. Goldman, D. K. Shumaker, and K. L. Wilson, 2005, "The nuclear lamina comes of age," Nat. Rev. Mol. Cell Biol. 6, 21. 
Haliloglu, T., I. Bahar, and B. Erman, 1997, "Gaussian dynamics of folded proteins," Phys. Rev. Lett. 79, 3090.

Han, S. S., A. C. T. van Duin, W. A. Goddard, and H. M. Lee, 2005, "Optimization and application of lithium parameters for the reactive force field, ReaxFF," J. Phys. Chem. A 109, 4575.

Hardy, J., and D. J. Selkoe, 2002, "Medicine-The amyloid hypothesis of Alzheimer's disease: Progress and problems on the road to therapeutics," Science 297, 353.

Hayward, S., and N. Go, 1995, "Collective variable description of native protein dynamics," Annu. Rev. Phys. Chem. 46, 223.

Heymann, B., and H. Grubmuller, 2000, "Dynamic force spectroscopy of molecular adhesion bonds," Phys. Rev. Lett. 84, 6126.

Holland, D., and M. Marder, 1999, "Cracks and atoms," Adv. Mater. 11, 793.

Hwang, W., S. G. Zhang, R. D. Kamm, and M. Karplus, 2004, "Kinetic control of dimer structure formation in amyloid fibrillogenesis," Proc. Natl. Acad. Sci. U.S.A. 101, 12916.

Hyeon, C., and D. Thirumalai, 2007, "Measuring the energy landscape roughness and the transition state location of biomolecules using single molecule mechanical unfolding experiments," J. Phys.: Condens. Matter 19, 113101.

Iconomidou, V. A., and S. J. Hamodrakas, 2008, "Natural protective amyloids," Curr. Protein Pept. Sci. 9, 291.

Irwin, G. R., 1957, "Analysis of stresses and strains near the end of a crack traversing a plate," J. Appl. Mech. 35, 379.

Keten, S., and M. J. Buehler, 2008a, “Asymptotic strength limit of hydrogen bond assemblies in proteins at vanishing pulling rates," Phys. Rev. Lett. 100, 198301.

Keten, S., and M. J. Buehler, 2008b, "Geometric confinement governs the rupture strength of H-bond assemblies at a critical length scale," Nano Lett. 8, 743.

Keten, S., and M. J. Buehler, 2008c, "Strength limit of entropic elasticity in beta-sheet protein domains," Phys. Rev. E 78, 061913.

Keten, S., Z. Xu, N. B. Ihle, and M. J. Buehler, 2010, "Nanoconfinement controls stiffness, strength, and mechanical toughness of $\beta$-sheet crystals in silk," Nature Mater. 9, 359.

Knowles, T. P., A. W. Fitzpatrick, S. Meehan, H. R. Mott, M. Vendruscolo, C. M. Dobson, and M. E. Welland, 2007, "Role of intermolecular forces in defining material properties of protein nanofibrils," Science 318, 1900.

Koester, K. J., J. W. Ager, and R. O. Ritchie, 2008, "The true toughness of human cortical bone measured with realistically short cracks," Nature Mater. 7, 672.

Kushima, A., X. Lin, J. Li, J. Eapen, J. C. Mauro, X. F. Qian, P. Diep, and S. Yip, 2009, "Computing the viscosity of supercooled liquids," J. Chem. Phys. 130, 224504.

Laioa, A., and M. Parrinello, 2002, "Escaping free-energy minima," Proc. Natl. Acad. Sci. U.S.A. 99, 12562.

Lakes, R., 1993, "Materials with structural hierarchy," Nature (London) 361, 511.

Lammerding, J., P. C. Schulze, T. Takahashi, S. Kozlov, T. Sullivan, R. D. Kamm, C. L. Stewart, and R. T. Lee, 2004, "Lamin A/C deficiency causes defective nuclear mechanics and mechanotransduction," J. Clin. Invest. 113, 370.

Lau, T. T., A. Kushima, and S. Yip, 2009, "An atomistic method for slow structural deformations," IOP Conf. Ser.: Mater. Sci. Eng. 3, 012002.

Lee, E. H., M. Gao, N. Pinotsis, M. Wilmanns, and K. Schulten, 2006, "Mechanical strength of the titin Z1Z2telethonin complex," Structure (London) 14, 497.
Lu, H., and K. Schulten, 2000, "The key event in force-induced unfolding of titin's immunoglobulin domains," Biophys. J. 79, 51.

Mackerell, A. D., 2004, "Empirical force fields for biological macromolecules: Overview and issues," J. Comput. Chem. 25, 1584.

MacKerell, A. D., D. Bashford, M. Bellott, R. L. Dunbrack, J. D. Evanseck, M. J. Field, S. Fischer, J. Gao, H. Guo, S. Ha, D. Joseph-McCarthy, L. Kuchnir, K. Kuczera, F. T. K. Lau, C. Mattos, S. Michnick, T. Ngo, D. T. Nguyen, B. Prodhom, W. E. Reiher, B. Roux, M. Schlenkrich, J. C. Smith, R. Stote, J. Straub, M. Watanabe, J. Wiorkiewicz-Kuczera, D. Yin, and M. Karplus, 1998, "All-atom empirical potential for molecular modeling and dynamics studies of proteins," J. Phys. Chem. B 102, 3586.

Marrink, S. J., H. J. Risselada, S. Yefimov, D. P. Tieleman, and A. H. de Vries, 2007, "The MARTINI force field: Course grained model for biomolecular simulations," J. Phys. Chem. B 111, 7812 .

Mayo, S. L., B. D. Olafson, and W. A. Goddard, 1990, "DREIDING-A generic force-field for molecular simulations," J. Phys. Chem. 94, 8897.

McBride, D. J., V. Choe, J. R. Shapiro, and B. Brodsky, 1997, "Altered collagen structure in mouse tail tendon lacking the alpha2(I) chain,” J. Mol. Biol. 270, 275.

Mesquida, P., C. K. Riener, C. E. MacPhee, and R. A. McKendry, 2007, "Morphology and mechanical stability of amyloid-like peptide fibrils," J. Mater. Sci.: Mater. Med. 18, 1325.

Miller, A., D. Delos, T. Baldini, T. M. Wright, and N. P. Camacho, 2007, "Abnormal mineral-matrix interactions are a significant contributor to fragility in oim/oim bone," Calcif. Tissue Int. 81, 206.

Misof, K., W. J. Landis, K. Klaushofer, and P. Fratzl, 1997, "Collagen from the osteogenesis imperfecta mouse model (oim) shows reduced resistance against tensile stress," J. Clin. Invest. 100, 40.

Monticelli, L., S. K. Kandasamy, X. Periole, R. G. Larson, D. P. Tieleman, and S. J. Marrink, 2008, "The MARTINI coarsegrained force field: Extension to proteins," J. Chem. Theory Comput. 4, 819.

Mostaert, A. S., M. J. Higgins, T. Fukuma, F. Rindi, and S. P. Jarvis, 2006, "Nanoscale mechanical characterisation of amyloid fibrils discovered in a natural adhesive," J. Biol. Phys. Chem. 32, 393.

Nalla, R. K., J. H. Kinney, and R. O. Ritchie, 2003, "Mechanistic fracture criteria for the failure of human cortical bone," Nature Mater. 2, 164.

Nalla, R. K., J. J. Kruzic, J. H. Kinney, M. Balooch, J. W. Ager, and R. O. Ritchie, 2006, "Role of microstructure in the agingrelated deterioration of the toughness of human cortical bone," Mater. Sci. Eng., C 26, 1251.

Nalla, R. K., J. S. Stolken, J. H. Kinney, and R. O. Ritchie, 2005, "Fracture in human cortical bone: local fracture criteria and toughening mechanisms," J. Biomech. 38, 1517.

Nelson, M. T., W. Humphrey, A. Gursoy, A. Dalke, L. V. Kale, R. D. Skeel, and K. Schulten, 1996, "NAMD: A parallel, object oriented molecular dynamics program," Int. J. Supercomput. Appl. 10, 251.

Neri, M., C. Anselmi, M. Cascella, A. Maritan, and P. Carloni, 2005, "Coarse-grained model of proteins incorporating atomistic detail of the active site," Phys. Rev. Lett. 95, 218102.

Nguyen, H., and C. Hall, 2004, "Molecular dynamics simula- 
tions of spontaneous fibril formation by random-coil peptides," Proc. Natl. Acad. Sci. U.S.A. 101, 16180.

Nguyen, H., and C. Hall, 2006, "Spontaneous fibril formation by polyalanines; Discontinuous molecular dynamics simulations," J. Am. Chem. Soc. 128, 1890.

Nielson, K. D., A. C. T. v. Duin, J. Oxgaard, W. Deng, and W. A. Goddard, 2005, "Development of the ReaxFF reactive force field for describing transition metal catalyzed reactions, with application to the initial stages of the catalytic formation of carbon nanotubes," J. Phys. Chem. A 109, 493.

Oberhauser, A. F., P. E. Marszalek, H. P. Erickson, and J. M. Fernandez, 1998, "The molecular elasticity of the extracellular matrix protein tenascin," Nature (London) 393, 181.

Pearlman, D. A., D. A. Case, J. W. Caldwell, W. S. Ross, T. E. Cheatham, S. Debolt, D. Ferguson, G. Seibel, and P. Kollman, 1995, "Amber, a package of computer-programs for applying molecular mechanics, normal-mode analysis, molecular-dynamics and free-energy calculations to simulate the structural and energetic properties of molecules," Comput. Phys. Commun. 91, 1.

Pellenq, R., A. Kushimac, R. Shahsavari, K. J. V. Van Vliet, M. J. Buehler, S. Yip, and F.-J. Ulm, 2009, “A realistic molecular model of cement hydrates," Proc. Natl. Acad. Sci. U.S.A. 106, 16102.

Penel, S., R. G. Morrison, P. D. Dobson, R. J. MortishireSmith, and A. J. Doig, 2003, "Length preferences and periodicity in beta-strands. Antiparallel edge beta-sheets are more likely to finish in non-hydrogen bonded rings," Protein Eng. 16, 957.

Ponder, J., and D. Case, 2003, "Force fields for protein simulations," Protein Simul. 66, 27.

Prockop, D. J., and K. I. Kivirikko, 1995, “Collagens: Molecular biology, diseases, and potentials for therapy," Annu. Rev. Biochem. 64, 403.

Qin, Z., L. Kreplak, and M. J. Buehler, 2009a, "Hierarchical structure controls nanomechanical properties of vimentin intermediate filaments," PLoS ONE 4, e7294.

Qin, Z., L. Kreplak, and M. J. Buehler, 2009b, "Nanomechanical properties of vimentin intermediate filament dimers," Nanotechnology 20, 425101.

Rappe, A. K., C. J. Casewit, K. S. Colwell, W. A. Goddard, and W. M. Skiff, 1992, "Uff, a full periodic-table force-field for molecular mechanics and molecular-dynamics simulations," J. Am. Chem. Soc. 114, 10024.

Rauch, F., and F. H. Glorieux, 2004, "Osteogenesis imperfecta," Lancet 363, 1377.

Reches, M., and E. Gazit, 2007, in Peptide Nanomaterials: SelfAssembling Peptides as Building Blocks for Novel Materials. Nanomaterials Chemistry: Novel Aspects and New Directions, edited by C. N. R. Rao, A. Mueller, and A. K. Cheetham (Wiley, New York), p. 171.

Rief, M., M. Gautel, F. Oesterhelt, J. M. Fernandez, and H. E. Gaub, 1997, "Reversible unfolding of individual titin immunoglobulin domains by AFM," Science 276, 1109.

Rief, M., M. Gautel, A. Schemmel, and H. E. Gaub, 1998, "The mechanical stability of immunoglobulin and fibronectin III domains in the muscle protein titin measured by atomic force microscopy," Biophys. J. 75, 3008.

Rief, M., J. Pascual, M. Saraste, and H. E. Gaub, 1999, "Single molecule force spectroscopy of spectrin repeats: Low unfolding forces in helix bundles," J. Mol. Biol. 286, 553.

Ritchie, R. O., M. J. Buehler, and P. Hansma, 2009, "Plasticity and toughness in bone: An issue of multiple length-scales,"
Phys. Today 62, 41.

Rohs, R., C. Etchebest, and R. Lavery, 1999, "Unraveling proteins: A molecular mechanics study," Biophys. J. 76, 2760.

Rosa, A., T. X. Hoang, D. Marenduzzo, and A. Maritan, 2003, "Elasticity of semiflexible polymers with and without selfinteractions," Macromolecules 36, 10095.

Rosakis, A. J., 2002, "Intersonic shear cracks and fault ruptures," Adv. Phys. 51, 1189.

Rosakis, A. J., H. Kanamori, and K. Xia, 2006, "Laboratory earthquakes," Int. J. Fract. 138, 211.

Rosakis, A. J., O. Samudrala, and D. Coker, 1999, "Cracks faster than the shear wave speed," Science 284, 1337.

Rountree, C. L., R. K. Kalia, E. Lidorikis, A. Nakano, L. v. Brutzel, and P. Vashishta, 2002, "Atomistic aspects of crack propagation in brittle materials: Multimillion atom molecular dynamics simulations," Annu. Rev. Mater. Res. 32, 377.

Sanbonmatsu, K. Y., and C. S. Tung, 2007, "High performance computing in biology: Multimillion atom simulations of nanoscale systems," J. Struct. Biol. 157, 470.

Scheraga, H. A., M. Khalili, and A. Liwo, 2007, "Proteinfolding dynamics: Overview of molecular simulation techniques," Annu. Rev. Phys. Chem. 58, 57.

Seifert, U., 2000, "Rupture of multiple parallel molecular bonds under dynamic loading," Phys. Rev. Lett. 84, 2750.

Sekiguchi, H., and T. Iwata, 2002, "Rupture process of the 1999 Kocaeli, Turkey, earthquake estimated from strongmotion waveforms," Bull. Seismol. Soc. Am. 92, 300.

Selkoe, D. J., 2001, “Alzheimer's disease: Genes, proteins, and therapy," Physiol. Rev. 81, 741.

Sims, T. J., C. A. Miles, A. J. Bailey, and N. P. Camacho, 2003, "Properties of collagen in OIM mouse tissues," Connect. Tissue Res. 44, 202.

Smith, J. F., T. P. J. Knowles, C. M. Dobson, C. E. MacPhee, and M. E. Welland, 2006, "Characterization of the nanoscale properties of individual amyloid fibrils," Proc. Natl. Acad. Sci. U.S.A. 103, 15806.

Sotomayor, M., and K. Schulten, 2007, "Single-molecule experiments in vitro and in silico," Science 316, 1144.

Strachan, A., E. M. Kober, A. C. T. van Duin, J. Oxgaard, and W. A. Goddard, 2005, "Thermal decomposition of RDX from reactive molecular dynamics," J. Chem. Phys. 122, 054502.

Strachan, A., A. C. T. van Duin, D. Chakraborty, S. Dasgupta, and W. A. Goddard, 2003, "Shock waves in high-energy materials: The initial chemical events in nitramine RDX," Phys. Rev. Lett. 91, 098301.

Stuart, S. J., A. B. Tutein, and J. A. Harrison, 2000, "A reactive potential for hydrocarbons with intermolecular interactions," J. Chem. Phys. 112, 6472.

Sulkowska, J. I., and M. Cieplak, 2007, "Mechanical stretching of proteins-a theoretical survey of the Protein Data Bank," J. Phys.: Condens. Matter 19, 283201.

Taylor, D., J. G. Hazenberg, and T. C. Lee, 2007, "Living with cracks: Damage and repair in human bone," Nature Mater. 6, 263.

Tirion, M., 1996, "Large amplitude elastic motions in proteins from a single-parameter, atomic analysis," Phys. Rev. Lett. 77, 1905.

Tozzini, V., 2005, "Coarse-grained models for proteins," Curr. Opin. Struct. Biol. 15, 144.

Van der Spoel, D., E. Lindahl, B. Hess, G. Groenhof, A. Mark, and H. Berendsen, 2005, "GROMACS: Fast, flexible, and free," J. Comput. Chem. 26, 1701.

van Duin, A. C. T., K. Nielson, W. Q. Deng, J. Oxgaard, and 
W. A. Goddard, 2004, "Application of ReaxFF reactive force fields to transition metal catalyzed nanotube formation," Abstr. Pap. - Am. Chem. Soc. 227, U1031.

Vashishta, P., R. K. Kalia, and A. Nakano, 1999, "Large-scale atomistic simulations of dynamic fracture," Comput. Sci. Eng. 1,56 .

Vollrath, F., and D. P. Knight, 2001, "Liquid crystalline spinning of spider silk," Nature (London) 410, 541.

Voter, A. F., F. Montalenti, and T. C. Germann, 2002, "Extending the time scale in atomistic simulation of materials," Annu. Rev. Mater. Res. 32, 321.

Wang, N., and D. Stamenovic, 2002, "Mechanics of vimentin intermediate filaments," J. Muscle Res. Cell Motil. 23, 535.

Wang, W., O. Donini, C. M. Reyes, and P. A. Kollman, 2001, "Biomolecular simulations: Recent developments in force fields, simulations of enzyme catalysis, protein-ligand, protein-protein, and protein-nucleic acid noncovalent interactions," Annu. Rev. Biophys. Biomol. Struct. 30, 211.
Weiner, S., and H. D. Wagner, 1998, "The material bone: Structure mechanical function relations," Annu. Rev. Mater. Sci. 28, 271.

West, D. K., D. J. Brockwell, P. D. Olmsted, S. E. Radford, and E. Paci, 2006, "Mechanical resistance of proteins explained using simple molecular models," Biophys. J. 90, 287.

Xia, K. W., A. J. Rosakis, and H. Kanamori, 2004, "Laboratory earthquakes: The sub-Rayleigh-to-supershear rupture transition," Science 303, 1859.

Xia, K. W., A. J. Rosakis, H. Kanamori, and J. R. Rice, 2005, "Laboratory earthquakes along inhomogeneous faults: Directionality and supershear," Science 308, 681.

Yung, Y. C., J. Chae, M. J. Buehler, C. Hunter, and D. Mooney, 2009, "Cyclic tensile strain triggers a sequence of autocrine and paracrine signaling to regulate angiogenic sprouting in human vascular cells," Proc. Natl. Acad. Sci. U.S.A. 106, 15279. 